\title{
Good deal hedging and valuation under combined uncertainty about drift and volatility
}

\author{
Dirk Becherer (D) . Klebert Kentia
}

Received: 8 April 2017 / Accepted: 9 November 2017 / Published online: 29 December 2017 (C) The Author(s). 2017 Open Access This article is distributed under the terms of the Creative Commons Attribution 4.0 International License (http://creativecommons.org/licenses/by/4.0/), which permits unrestricted use, distribution, and reproduction in any medium, provided you give appropriate credit to the original author(s) and the source, provide a link to the Creative Commons license, and indicate if changes were made.

\begin{abstract}
We study robust notions of good-deal hedging and valuation under combined uncertainty about the drifts and volatilities of asset prices. Good-deal bounds are determined by a subset of risk-neutral pricing measures such that not only opportunities for arbitrage are excluded but also deals that are too good, by restricting instantaneous Sharpe ratios. A non-dominated multiple priors approach to model uncertainty (ambiguity) leads to worst-case good-deal bounds. Corresponding hedging strategies arise as minimizers of a suitable coherent risk measure. Good-deal bounds and hedges for measurable claims are characterized by solutions to secondorder backward stochastic differential equations whose generators are non-convex in the volatility. These hedging strategies are robust with respect to uncertainty in the sense that their tracking errors satisfy a supermartingale property under all a-priori valuation measures, uniformly over all priors.
\end{abstract}

Keywords Combined drift and volatility uncertainty $\cdot$ Good-deal bounds $\cdot$ Robust hedging $\cdot$ Hedging to acceptability $\cdot$ Second-order BSDE $\cdot$ Stochastic control

Mathematics Subject Classification (2000) 60G44 • 60H30 • 91G10 • 93E20 • 91B06 $\cdot 91 \mathrm{~B} 30$

\footnotetext{
D. Becherer $(\bowtie)$

Institut für Mathematik, Humboldt Universität, Unter den Linden 6, 10099 Berlin, Germany

e-mail: becherer@mathematik.hu-berlin.de

K. Kentia

Institut für Mathematik, Goethe-Universität, D-60054 Frankfurt am Main, Germany

e-mail: kentia@aims.ac.za
} 


\section{Introduction}

Hedging and valuation under model uncertainty (ambiguity) about volatility has been a seminal problem in the topical area of robust finance. In mathematics, it has motivated to no small extend recent advances on subjects such as second-order backward stochastic differential equations (2BSDEs), G-expectations and related stochastic calculus, sub-linear conditional expectations, and control of non-linear kernels, using a variety of different methods from stochastic control, quasi-sure analysis and capacity theory, or expectation-spaces and PDE-theory, see Denis and Martini (2006), Peng (2007), Denis et al. (2011), Nutz (2012b), Nutz and Soner (2012), Soner et al. (2012), Nutz and van Handel (2013), Hu et al. (2014a), Possamaï et al. (2018) and many more references therein. The research has been challenging (and fruitful) since one has to deal (in a probabilistic setup) with families of non-dominated probability measures, also called multiple priors, that can be mutually singular. In contrast, uncertainty solely about drifts in a continuous time setting of stochastic Itô-processes could be dealt with in a dominated framework of measures which are absolutely continuous with respect to a single reference probability measure.

The main contributions of the current paper are twofold. For incomplete markets in continuous time, we solve the problem of robust hedging and valuation under combined uncertainty about both the drifts and the volatilities of the Itô processes which describe the evolution of the tradeable asset prices in an underlying non-Markovian model for the financial market. Further, we investigate to this end the no-good-deal approach to hedging and valuation, that is much cited in the finance literature (cf. Cochrane and Saá-Requejo (2000), Cerný and Hodges (2002) or Björk and Slinko (2006)) and provides more narrow valuation bounds and less extreme hedges than the more fundamental approach of almost-sure-hedging by superreplication with its corresponding no-arbitrage valuation bounds.

Concerning the influential application of hedging under volatility uncertainty in continuous-time models which has been stipulated, at least, by Avellaneda et al. (1995) and Lyons (1995), the literature so far has almost entirely been concerned with the superreplication approach, for uncertainty being restricted solely to volatility as ambiguity about the drift of the asset prices has no effect there. While the notion of superreplication is fundamental to the theory of stochastic processes and for applications, being related to the optional decomposition and excluding the possibility of losses, it is also known from a practical point of view that superreplication could be overly expensive already in the absence of uncertainty for incomplete market models. This calls for an adaption of other, less conservative, concepts for partial (not almost-sure) hedging in incomplete markets to a framework that is robust with respect to model ambiguity. Under combined uncertainties on drift and volatility, which are going to be relevant to such alternative approaches where the (ambiguous) distributions of hedging errors matter, however new mathematical challenges have to be overcome, as the non-dominated family of measures will not just consist of local martingale measures, like for instance in Soner et al. (2012). Such has been noted and addressed just recently in Nutz (2012b), Epstein and Ji (2014) and Possamaï et al. (2018). Likewise, we are aware of only few recent articles on the related problem of expected utility maximization under uncertainty about both 
drifts and volatilities (cf. Tevzadze et al 2013; Biagini and Pinar 2017; Neufeld and Nutz 2016) some of which achieve quite explicit results for models with specific parametric structure. Among many interesting contributions on utility optimization under only one type of uncertainty, see for instance Chen and Epstein (2002), Quenez (2004), Garlappi et al. (2007), Schied (2007) or Øksendal and Sulem (2014) for (dominated) uncertainty solely about drifts, or Matoussi et al. (2015) and Hu et al. (2014b) for ambiguity solely about volatilities but not about drifts. For equilibrium prices of a representative agent under ambiguity about the volatility, see Epstein and Ji (2013, Section 3.3). To the best of our knowledge, there appear to be hardly any studies yet on hedging approaches for (generically) incomplete markets under combined ambiguity about drifts and volatilities - apart from superreplication.

We are going to investigate a robust extension of the no-good-deal hedging approach in continuous time under combined ambiguity about the volatilities and drifts. Without model uncertainty, good-deal bounds have been introduced as valuation bounds in incomplete markets which do not only prevent opportunities for arbitrage but also for deals with an overly attractive reward-for-risk ratio. The most cited reference in the finance literature appears to be Cochrane and Saá-Requejo (2000). We refer to Björk and Slinko (2006), Klöppel and Schweizer (2007) and Cerný and Hodges (2002) for mathematical and conceptual ideas and many more references. By using only a suitable subset of "no-good-deal" risk neutral prices, the resulting valuation bounds are tighter than the classical no-arbitrage bounds (which are often too wide) but still have economic meaning. Good-deal bounds have been defined predominantly by constraints on the instantaneous Sharpe ratios in (any) extension of the financial market by additional derivatives' price processes, see Björk and Slinko (2006). For models without jumps, such is equivalent to imposing constraints on the optimal expected growth rates, cf. Becherer (2009). Although good-deal theory has been initiated merely as a valuation approach (cf. the conclusions in Björk and Slinko 2006), a corresponding notion of (good-deal) hedging strategies can be defined as minimizers of a suitable dynamic coherent risk measure, in the spirit of Barrieu and El Karoui (2009), such that the good-deal bounds appear as market consistent risk measures, cf. Becherer (2009). We would like to mention that this is closely related to the idea of pricing and hedging to acceptability that has been put forward in the realm of conic finance, see Madan and Cherny (2010) and Remark 10. It has to be noted, however, that the corresponding theoretical results on valuations and hedges are sensitive to the assumptions of the probability model about the dynamics of the asset prices. Since the objective real world probability measure is not precisely known and financial models can, at best, be useful but idealized simplifications of reality, robust approaches to model ambiguity are relevant to advance the theory.

To our best knowledge, a robust approach to good-deal hedging in continuous time under non-dominated uncertainty has not been available so far. For drift uncertainty and more references, see Becherer and Kentia (2017). Robust results on valuation and hedging will be obtained by $2 \mathrm{BSDE}$ theory, building on recent advances by Possamaï et al. (2018) whose analysis provides a general wellposedness result that fits well to our application with combined uncertainty, cf. Remark 12. Indeed, their result neither requires convexity nor uniform continuity of the generator, and it can 
deal with general (measurable) contingent claims without assuming further regularity (like e.g. uniform continuity) or a Markovian framework.

The organization of the present paper is as follows. The setup and preliminaries are explained in the Section "Mathematical framework and preliminaries", with a brief summary of key results on 2BSDEs. Then we begin the Section "Financial market model and good-deal constraints" by a description of the financial market and the (non-dominated) confidence set of (uncertain) priors that captures the combined ambiguity about drifts and volatilities. In comparison to most literature on hedging under ambiguous volatility, like Avellaneda et al. (1995) or Lyons (1995), we are going to consider a model for asset prices that constitutes a generically incomplete market, even if seen just under (any) one individual prior. That means not only that there exists, in general, no perfect hedging (i.e. replicating) strategy which is robust with respect to ambiguity on priors, but that in general there might not even exists a replicating strategy in the model with (any) one given probability prior, even without ambiguity. Section "Financial market model and good-deal constraints" then proceeds by taking drift and volatility to be known at first, in order to explain the idea for the good-deal approach as simply as possible. Following classical good-deal theory, good-deal restrictions are defined by constraints on the instantaneous Shape ratios, i.e., by radial bounds on the Girsanov kernels of pricing measures, and standard BSDE descriptions of valuation bounds and hedges are summarized. Adopting a multiple-priors approach, like, e.g., in Gilboa and Schmeidler (1989) or Chen and Epstein (2002), while accommodating for the fact that priors here are non-dominated, Section "Good-deal hedging and valuation under combined uncertainty" starts by defining the good-deal bounds under uncertainty as the worst-case bounds over all priors. For hedging purposes, we define good-deal hedging strategies as minimizers of suitable a-priori risk measures under optimal risk sharing with the market. We derive 2BSDE characterizations for the dynamic valuation bounds and the hedging strategies. We show that tracking errors from good-deal hedging satisfy a supermartingale property under all a-priori valuation measures uniformly for all priors. In contrast to the situation without volatility uncertainty, where classical wellposedness and comparison theorems for BSDEs are used by Becherer and Kentia (2017) to derive characterizations of valuations and hedges by standard BSDE, the case including volatility uncertainty poses more technical difficulties in using 2BSDEs. Indeed, the wellposedness and comparison for 2BSDEs require more stringent measurability and integrability conditions on the generators and terminal data to be satisfied in terms of the additional parameter of volatility. Under combined uncertainty, the proof to the valuation 2BSDE (cf. Theorem 1) relies on measurable selection arguments to show the required conditions on the generator. Furthermore, the proof for the characterization of hedging strategies by 2BSDEs (cf. Theorem 2) instead uses saddle-point arguments (cf. Lemma 4) to identify the robust good-deal hedging strategy through a minmax identity. To illustrate the extend of our results, we complete Section "Good-deal hedging and valuation under combined uncertainty" with a simple but instructive example about hedging a put option on a non-traded (but correlated) asset in an incomplete market. This allows for some elementary closed form solutions, offering intuition for the general but abstract main Theorem 2. For instance, it illustrates that the good-deal hedging strategy generally is very different from the 
super-replicating strategy, which has been studied in e.g. Avellaneda et al. (1995), Lyons (1995), Denis and Martini (2006), Nutz and Soner (2012), Neufeld and Nutz (2013), Vorbrink (2014). The concrete case study also illustrates, how already in an elementary Markovian example additional complications arise from combined uncertainty.

\section{Mathematical framework and preliminaries}

We consider filtered probability space $\left(\boldsymbol{\Omega}, \mathcal{F}=\mathcal{F}_{T}, P^{0}, \mathbb{F}\right)$, where $\boldsymbol{\Omega}:=\{\omega \in$ $\left.\mathcal{C}\left([0, T], \mathbb{R}^{n}\right): \omega(0)=0\right\}$ is the canonical space of continuous paths starting at 0 endowed with the norm $\|\omega\|_{\infty}:=\sup _{t \in[0, T]}|\omega(t)|$. The filtration $\mathbb{F}=\left(\mathcal{F}_{t}\right)_{t \in[0, T]}$ is generated by the canonical process $B_{t}(\omega):=\omega(t), \omega \in \boldsymbol{\Omega}$, and $P^{0}$ is the Wiener measure. We denote by $\mathbb{F}_{+}=\left(\mathcal{F}_{t}^{+}\right)_{t \in[0, T]}$ the right-limit of $\mathbb{F}$, with $\mathcal{F}_{t}^{+}=\mathcal{F}_{t+}:=$ $\cap_{s>t} \mathcal{F}_{s}$. For a probability measure $Q$, the conditional expectation given $\mathcal{F}_{t}$ will be denoted by $E_{t}^{Q}[\cdot]$. A probability measure $P$ is called a local martingale measure if $B$ is a local martingale w.r.t. $(\mathbb{F}, P)$. One can, cf. Karandikar (1995), construct the quadratic variation process $\langle B\rangle$ pathwise such that it coincides with $\langle B\rangle^{P} P$-a.s. for all local martingale measures $P$. In particular, this yields a pathwise definition of the density $\widehat{a}$ of $\langle B\rangle$ w.r.t. the Lebesgue measure as

$$
\widehat{a}_{t}(\omega):=\limsup _{\epsilon \searrow 0} \frac{1}{\epsilon}\left(\langle B\rangle_{t}(\omega)-\langle B\rangle_{t-\epsilon}(\omega)\right), \quad(t, \omega) \in[0, T] \times \Omega .
$$

We define by $\mathbb{S}_{n}^{>0} \subset \mathbb{R}^{n \times n}$ the space of positive definite symmetric $n \times n$-matrices. In the sequel, for any matrix $A \in \mathbb{S}_{n}^{>0}$ we denote by $A^{\frac{1}{2}}$ the unique matrix in $\mathbb{S}_{n}^{>0}$ such that $A^{\frac{1}{2}} A^{\frac{1}{2}}=A$. The matrix $A^{\frac{1}{2}}$ is called the (principal) square root of $A$ and the operation $\mathbb{S}_{n}^{>0} \ni A \mapsto A^{\frac{1}{2}} \in \mathbb{S}_{n}^{>0}$ is continuous.

Remark 1 In place of the (symmetric) principal square root for matrices in $\mathbb{S}_{n}^{>0}$, other fixed choices of (continuous) square root operations (not neccessarily symmetric or positive definite) would be possible too, e.g. as in (Possamaï et al. 2018), at some notational expenses of replacing at several places square root matrices by their transposes or the natural matrix inverse by a suitable pseudo-inverse. In general, square root operations might fail to be continuous on the space of matrices. However, our choosen symmetric principal square root operation is continuous, what is used to apply measurable selection theorems in the proof of Theorem 1.

We denote by $\overline{\mathcal{P}}_{W}$ the set of all local martingale measures $P$ for which $\widehat{a}$ is well-defined and takes values in $\mathbb{S}_{n}^{>0} P$-a.e.. Note that the measures in $\overline{\mathcal{P}}_{W}$ could be mutually singular, as noted in Soner et al. (2011). For any $P \in \overline{\mathcal{P}}_{W}$, the process $W^{P}:={ }^{(P)} \int_{0} \widehat{a}_{s}^{-\frac{1}{2}} d B_{S}$ is a Brownian motion under $P$. To formulate volatility uncertainty, we concentrate only on the subclass $\overline{\mathcal{P}}_{S} \subset \overline{\mathcal{P}}_{W}$ of measures

$$
P^{\alpha}:=P^{0} \circ\left(X^{\alpha}\right)^{-1}, \quad \text { where } X^{\alpha}:={ }^{\left(P^{0}\right)} \int_{0}^{\cdot} \alpha_{s}^{1 / 2} d B_{s},
$$


with $\mathbb{S}_{n}^{>0}$-valued $\mathbb{F}$-progressively measurable $\alpha$ satisfying $\int_{0}^{T}\left|\alpha_{t}\right| d t<\infty, P^{0}$-a.s.. A benefit of restricting to the subclass $\overline{\mathcal{P}}_{S}$ is the following aggregation property (cf. Soner et al. 2011, Lemma 8.1, Lemma 8.2).

Lemma 1 For $P \in \overline{\mathcal{P}}_{W}$, let $\mathbb{F}^{P}$ denote the $P$-augmentation of the filtration $\mathbb{F}$ and $\overline{\mathbb{F}}^{W^{P}}$ the one of the natural filtration $\mathbb{F}^{W^{P}}$ for $W^{P}$. Then $B$ has the martingale representation property w.r.t. $\left(\mathbb{F}^{P}, P\right)$ for all $P \in \overline{\mathcal{P}}_{S}$, and $\overline{\mathcal{P}}_{S}=$ $\left\{P \in \overline{\mathcal{P}}_{W}: \mathbb{F}^{P}=\overline{\mathbb{F}}^{W^{P}}\right\}$. Moreover, $(P, \mathbb{F})$ satisfies the Blumenthal zero-one law for any $P \in \overline{\mathcal{P}}_{S}$.

Remark 2 For any $P \in \overline{\mathcal{P}}_{S}$, Lemma 1 implies $E_{t}^{P}[X]=E^{P}\left[X \mid \mathcal{F}_{t}^{+}\right] P$-a.s. for any $X \in L^{1}(P), t \in[0, T]$. In particular, any $\mathcal{F}_{t}^{+}$-measurable random variable has a $\mathcal{F}_{t}$-measurable P-version.

Let $\underline{a}, \bar{a} \in \mathbb{S}_{n}^{>0}$. We will work with the subclass $\mathcal{P}_{[\underline{a}, \bar{a}]}$ of $\overline{\mathcal{P}}_{S}$ defined by

$$
\mathcal{P}_{[\underline{a}, \bar{a}]}:=\left\{P \in \overline{\mathcal{P}}_{S}: \underline{a} \leq \widehat{a} \leq \bar{a}, P \otimes d t \text {-a.e. }\right\}
$$

and assumed to be non-empty. We use the language of quasi-sure analysis as it appears in the framework of capacities of Denis and Martini (2006) as follows.

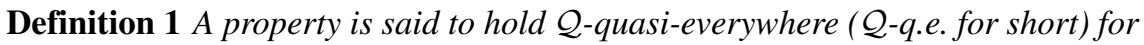
a family $\mathcal{Q}$ of measures on the same measurable space if it holds outside of a set, which is a nullset under each element of $\mathcal{Q}$.

Unless stated otherwise, inequalities between random variables will be meant in a $\mathcal{P}_{[\underline{a}, \bar{a}]}$-quasi-sure sense (written $\mathcal{P}_{[\underline{a}, \bar{a}]}$-q.s. for short), while inequalities between $\mathbb{F}_{+}$progressively measurable processes will be in the $\mathcal{P}_{[\underline{a}, \bar{a}]} \otimes d t$-q.e. sense, for $\mathcal{P}_{[\underline{a}, \bar{a}]} \otimes$ $d t:=\left\{P \otimes d t, P \in \mathcal{P}_{[\underline{a}, \bar{a}]}\right\}$. We now introduce spaces and norms of interest for the paper. Some of these spaces are already quite classical, and have been modified here to account for the possible mutual singularity of measures in $\mathcal{P}_{[\underline{a}, \bar{a}]}$. For a filtration $\mathbb{X}=\left(\mathcal{X}_{t}\right)_{t \in[0, T]}$ on $\left(\boldsymbol{\Omega}, \mathcal{F}_{T}\right)$ with augmentation $\mathbb{X}^{P}:=\left(\mathcal{X}_{t}^{P}\right)_{t \in[0, T]}$ under measure $P \in \mathcal{P}_{[\underline{a}, \bar{a}]}$, we consider the following function spaces:

a) $L_{\mathcal{P}_{[\underline{a}, \bar{a}]}}^{2}\left(\mathcal{X}_{T}\right)$ (resp., $\left.L^{2}\left(\mathcal{X}_{T}, P\right)\right)$ of $\mathcal{X}_{T}$-measurable real random variables $X$ with $\|X\|_{L_{\mathcal{P}_{[\underline{a}, \bar{a}]}^{2}}^{2}}^{2}=\sup _{P \in \mathcal{P}_{[\underline{a}, \bar{a}]}} E^{P}\left[|X|^{2}\right]<\infty\left(\right.$ resp., $\left.\|X\|_{L^{2}(P)}^{2}=E^{P}\left[|X|^{2}\right]<\infty\right)$,

b) $\mathbb{H}^{2}(\mathbb{X})$ (resp., $\mathbb{H}^{2}(\mathbb{X}, P)$ ) of $\mathbb{X}$-predictable $\mathbb{R}^{n}$-valued processes $Z$ with

$\|Z\|_{\mathbb{H}^{2}}^{2}=\sup _{P \in \mathcal{P}_{[\underline{a}, \bar{a}]}} E^{P}\left[\int_{0}^{T}\left|\widehat{a}_{t}^{\frac{1}{2}} Z_{t}\right|^{2} d t\right]<\infty\left(\right.$ resp., $\left.\|Z\|_{\mathbb{H}^{2}(P)}^{2}=E^{P}\left[\int_{0}^{T}\left|\widehat{a}_{t}^{\frac{1}{2}} Z_{t}\right|^{2} d t\right]<\infty\right)$, 
c) $\mathbb{D}^{2}(\mathbb{X})\left(\right.$ resp., $\left.\mathbb{D}^{2}(\mathbb{X}, P)\right)$ of all $\mathbb{X}$-progressively measurable $\mathbb{R}$-valued processes $Y$ with càdlàg paths $\mathcal{P}_{[\underline{a}, \bar{a}]}$-q.s. (resp., $P$-a.s.), and satisfying

$$
\|Y\|_{\mathbb{D}^{2}}:=\left\|\sup _{t \in[0, T]}\left|Y_{t}\right|\right\|_{L_{\mathcal{P}_{[a, \bar{a}]}^{2}}^{2}}<\infty\left(\operatorname{resp} .,\|Y\|_{\mathbb{D}^{2}(P)}:=\left\|\sup _{t \in[0, T]}\left|Y_{t}\right|\right\|_{L^{2}(P)}<\infty\right),
$$

d) $\mathbb{L}^{2}(\mathbb{X})$ the subspace of $L_{\mathcal{P}_{[\underline{a}, \bar{a}]}}^{2}\left(\mathcal{X}_{T}\right)$ consisting of random variables $X$ satisfying

$$
\|X\|_{\mathbb{L}^{2}}^{2}:=\sup _{P \in \mathcal{P}_{[\underline{a}, \bar{a}]}} E^{P}\left[\operatorname{ess}_{t \in[0, T]}^{P} \operatorname{ess}_{P^{\prime} \in \mathcal{P}_{[a, \bar{a}]}(t, P, \mathbb{X})}^{P} E^{P^{\prime}}\left[|X|^{2} \mid \mathcal{X} t\right]<\infty\right.
$$

for the set of measures $\mathcal{P}_{[\underline{a}, \bar{a}]}(t, P, \mathbb{X}):=\left\{P^{\prime} \in \mathcal{P}_{[\underline{a}, \bar{a}]}: P^{\prime}=P\right.$ on $\left.\mathcal{X}_{t}\right\}$,

e) $\mathbb{I}^{2}(\mathbb{X}, P)$ the space of $\mathbb{X}$-predictable processes $K$ with càdlàg and non-decreasing paths $P$-a.s., $K_{0}=0 P$-a.s., and $\|K\|_{\mathbb{I}^{2}(P)}^{2}:=E^{P}\left[K_{T}^{2}\right]<\infty$. In particular, we will denote by $\mathbb{I}^{2}\left(\left(\mathbb{X}^{P}\right)_{P \in \mathcal{P}_{[a, \bar{a}]}}\right)$ the family of tuples $\left(K^{P}\right)_{P \in \mathcal{P}_{[a, \bar{a}]}}$ such that $K^{P} \in$ $\mathbb{I}^{2}\left(\mathbb{X}^{P}, P\right)$ for any $P \in \mathcal{P}_{[\underline{a}, \bar{a}]}$ and $\sup _{P \in \mathcal{P}_{[a, \bar{a}]}}\left\|K^{P}\right\|_{\mathbb{I}^{2}(P)}<\infty$.

The reader will note the analogy with the spaces and norms defined in Possamaï et al. (2018) (though with slightly different notations) for the specific family of collection of measures $\mathcal{P}(t, \omega):=\mathcal{P}_{[\underline{a}, \bar{a}]}$ for any $(t, \omega) \in[0, T] \times \Omega$. A filtration which might in the sequel play the role of $\mathbb{X}$ in the definitions of spaces above is $\mathbb{F}^{\mathcal{P}_{[a, \bar{a}]}}=\left(\mathcal{F}_{t}^{\mathcal{P}_{[\underline{a}, \bar{a}]}}\right)_{t \in[0, T]}$, with $\mathcal{F}_{t}^{\mathcal{P}_{[a, \bar{a}]}}:=\bigcap_{P \in \mathcal{P}_{[\underline{a}, \bar{a}]}} \mathcal{F}_{t}^{P}, t \in[0, T]$.

\section{Second-order backward stochastic differential equations}

Following Possamaï et al. (2018), we summarize an existence and uniqueness result for Lipschitz 2BSDEs and state a representation of solutions that will be key to characterize the good-deal bounds and hedging strategies under combined drift and volatility uncertainties: See Proposition 1 . The generator for a $2 \mathrm{BSDE}$ is a function $F:[0, T] \times \boldsymbol{\Omega} \times \mathbb{R} \times \mathbb{R}^{n} \times \mathbb{S}_{n}^{>0} \rightarrow \mathbb{R}$ for which we denote $\widehat{F}_{t}(\omega, y, z):=F_{t}\left(\omega \cdot \wedge t, y, z, \widehat{a}_{t}(\omega)\right)$ and $\widehat{F}_{t}^{0}:=\widehat{F}_{t}(0,0)$. For wellposedness, we will require generators $F$ and terminal conditions $X$ to satisfy the following combination of Assumption 2.1.(i)-(ii) and Assumption 3.1. in Possamaï et al. (2018) (for $\kappa=p=2$ ).

\section{Assumption 1 (i) $X$ is $\mathcal{F}_{T}$-measurable,}

(ii) $F$ is jointly Borel measurable, and $\mathbb{F}$-progressive in $(t, \omega)$ for each $(y, z, a)$,

(iii) $\exists C>0$ such that for all $(t, \omega, a) \in[0, T] \times \Omega \times \mathbb{S}_{n}^{>0}, y, y^{\prime} \in \mathbb{R}, z, z^{\prime} \in \mathbb{R}^{n}$,

$$
\left|F_{t}(\omega, y, z, a)-F_{t}\left(\omega, y^{\prime}, z^{\prime}, a\right)\right| \leq C\left(\left|y-y^{\prime}\right|+\left|z-z^{\prime}\right|\right),
$$

(iv) $\widehat{F}^{0}$ satisfies $\left(\int_{0}^{T}\left|\widehat{F}_{s}^{0}\right|^{2} d s\right)^{1 / 2} \in \mathbb{L}^{2}\left(\mathbb{F}_{+}\right)$. 
Remark 3 Assumption 1(iv) is satisfied for F such that Assumption 1-(ii) holds and $\widehat{F}^{0}$ is bounded $\mathcal{P}_{[\underline{a}, \bar{a}]}$-q.s.. It implies $\sup _{P \in \mathcal{P}_{[\underline{a}, \bar{a}]}} E^{P}\left[\int_{0}^{T}\left|\widehat{F}_{s}^{0}\right|^{2} d s\right]<\infty$.

A second-order BSDE is a stochastic integral equation of the type

$$
Y_{t}=X-\int_{t}^{T} \widehat{F}_{s}\left(Y_{s}, \widehat{a}_{s}^{1 / 2} Z_{s}\right) d s-{ }^{(P)} \int_{t}^{T} Z_{s}^{\mathrm{tr}} d B_{s}+K_{T}^{P}-K_{t}^{P}, t \in[0, T], \mathcal{P}_{[\underline{a}, \bar{a}]-\text { q.s.. }}
$$

In comparison to Possamaï et al. (2018), because the canonical process $B$ satisfies the martingale representation property simultaneously under all measures in $\mathcal{P}_{[a, a]}$ (cf. Lemma 1), we do not have an orthogonal martingale components in the formulation of 2BSDEs as (2). The same formulation can be used in the more general framework with semimartingale laws for the canonical process, but working under a saturation property for the set of priors (cf. Possamaï et al. 2018, Definition 5.1).

Definition $2\left(Y, Z,\left(K^{P}\right)_{P \in \mathcal{P}_{[a, \bar{a}]}}\right) \in \mathbb{D}^{2}\left(\mathbb{F}^{\mathcal{P}_{[\underline{a}, \bar{a}]}}\right) \times \mathbb{H}^{2}\left(\mathbb{F}^{\left[\mathcal{P}_{[a, a]}\right]}\right) \times \mathbb{I}^{2}\left(\left(\mathbb{F}^{P}\right)_{P \in \mathcal{P}_{[\underline{a}, \bar{a}]}}\right)$ is called a solution (triple) to the $2 B S D E$ (2) if it satisfies the required dynamics $\mathcal{P}_{[\underline{a}, \bar{a}]^{-}-q . s . \text { and the family }}\left\{K^{P}, P \in \mathcal{P}_{[\underline{a}, \bar{a}]}\right\}$ satisfies the minimum condition

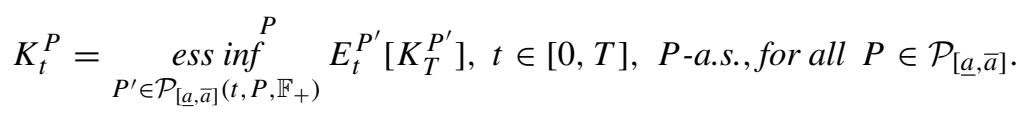

If the family $\left\{K^{P}, P \in \mathcal{P}_{[a, \bar{a}]}\right\}$ can be aggregated into a single process $K$, i.e., $K^{P}=K, P$-a.s. for all $P \in \mathcal{P}_{[\underline{a}, \bar{a}]}$, then $(Y, Z, K)$ is said to solve the $2 B S D E$.

Remark 4 Note in the 2BSDE dynamics (2) the dependence of the stochastic integrals ${ }^{(P)} \int_{0}^{\cdot} Z_{s}^{\text {tr }} d B_{s}$ on the probability measures $P \in \mathcal{P}_{[\underline{a}, \bar{a}]}$. Indeed, since the measures in $\mathcal{P}_{[a, \bar{a}]}$ may be non-dominated, it might be that these integrals do not aggregate (see Soner et al. 2011, for more on aggregation). However, under additional set theoretical assumptions (for instance, continuum hypothesis plus the axiom of choice) a method by Nutz (2012a) can be used to construct the stochastic integral $\int_{0}^{i} Z_{s}^{t r} d B_{s}$ pathwise for any predictable process $Z$. As a by-product, the family $\left\{K^{P}, P \in \mathcal{P}_{[\underline{a}, \bar{a}]}\right\}$ for a $2 B S D E$ solution $\left(Y, Z,\left(K^{P}\right)_{P \in \mathcal{P}_{[a, \bar{a}]}}\right)$ would automatically aggregate into a single process $K:=Y_{0}-Y+\int_{0} \widehat{F}_{s}\left(Y_{s}, \widehat{a}_{s}^{1 / 2} Z_{s}\right) d s+\int_{0}^{\cdot} Z_{s}^{t r} d B_{s}$ yielding a 2BSDE solution $(Y, Z, K)$. Reciprocally for a solution $(Y, Z, K)$, the family $\left\{{ }^{(P)} \int_{0}^{*} Z_{s}^{t r} d B_{s}, P \in \mathcal{P}_{[\underline{a}, \bar{a}]}\right\}$ also aggregates.

The pair $(F, X)$ will be called the parameters of the 2BSDE (2). We will refer to $Y$ as the value process and $Z$ as the control process. The following proposition provides the wellposedness result of interest in this paper, as well as a representation of the value process in terms of solutions to standard BSDEs. The proof relies on an application of (Possamaï et al. 2018, Theorem 4.1, Theorem 4.2) for the specific family of measures $\mathcal{P}_{[\underline{a}, \bar{a}]}$, the details being deferred to the Appendix. We employ 
the classical sign convention for standard BSDE generators according to which the generator of the BSDE (5) below is $-\widehat{F}$ (i.e., with a minus sign). The sign convention for a $2 \mathrm{BSDE}$ generator however remains as already introduced, e.g., the 2BSDE (2) has the generator $F$.

Proposition 1 Given $X \in \mathbb{L}^{2}\left(\mathbb{F}_{+}\right)$and Assumption 1, the 2BSDE (2) has a 1. unique solution $\left(Y, Z,\left(K^{P}\right)_{P \in \mathcal{P}_{[\underline{a}, \bar{a}]}}\right) \in \mathbb{D}^{2}\left(\mathbb{F}^{\mathcal{P}_{[\underline{a}, \bar{a}]}}\right) \times \mathbb{H}^{2}\left(\mathbb{F}^{\mathcal{P}_{[\underline{a}, \bar{a}]}}\right) \times$ $\mathbb{I}^{2}\left(\left(\mathbb{F}^{P}\right)_{P \in \mathcal{P}_{[\underline{a}, \bar{a}]}}\right)$,

2. and for any $P \in \mathcal{P}_{[\underline{a}, \bar{a}]}$, the $Y$-part of the solution has the representation

$$
Y_{s}=\underset{P^{\prime} \in \mathcal{P}_{[\underline{a}, \bar{a}]}\left(s, P, \mathbb{F}_{+}\right)}{\operatorname{ess} \sup _{s}^{P}} \mathcal{Y}_{s}^{P^{\prime}}\left(t, Y_{t}\right), s \leq t \leq T, P \text {-a.s. }
$$

where $\left(\mathcal{Y}^{P}(\tau, H), \mathcal{Z}^{P}(\tau, H)\right)$ denotes the unique solution to the standard BSDE

$$
\mathcal{Y}_{t}^{P}=H-\int_{t}^{\tau} \widehat{F}_{s}\left(\mathcal{Y}_{s}^{P}, \widehat{a}_{s}^{1 / 2} \mathcal{Z}_{s}^{P}\right) d s-{ }^{(P)} \int_{t}^{\tau}\left(\mathcal{Z}_{s}^{P}\right)^{t r} d B_{s}, t \leq \tau, P \text {-a.s. }
$$

with parameters $(-\widehat{F}, H)$, for an $\mathbb{F}^{P}$-stopping time $\tau$ and $H \in L^{2}\left(\mathcal{F}_{\tau}^{P}, P\right)$.

Remark 5 Note, in comparison to Soner et al. (2012), that uniform continuity (in $(\omega)$ ) and convexity (in a) of the generator function $F$ are not required for the more general wellposedness results of Possamaï et al. (2018) summarized here in Proposition 1. The latter is what we need in Section "Good-deal hedging and valuation under combined uncertainty" for applications to valuation and hedging under combined drift and volatility uncertainty, as the results of Soner et al. (2012) may not be applicable in that situation; cf. Part 1 of Remark 12 for a detailed justification. Note that the generalized theory also works for terminal conditions which are merely Borel measurable and do not need to be in the closure of uniformly continuous functions as required in Soner et al. (2012).

\section{Financial market model and good-deal constraints}

We apply the 2BSDE theory of Section "Second-order backward stochastic differential equations" to good-deal valuation and hedging of contingent claims in incomplete financial markets under drift and volatility uncertainty. In comparison to standard BSDEs which are used in Becherer and Kentia (2017) in the presence of solely drift uncertainty, 2BSDEs are an appropriate tool for describing worst-case valuations in the presence of volatility uncertainty. As in Cochrane and Saá-Requejo (2000); Björk and Slinko (2006), we consider good-deal constraints imposed as bounds on the Sharpe ratios (equivalently bounds on the optimal growth rates as in (Becherer 2009), ) in the financial market extended by further (derivative) asset price processes. But first we specify the model for the market with uncertainty about the volatility and the market price of risk. 


\section{Financial market with combined uncertainty about drift and volatility}

The financial market consists of $d$ tradeable stocks $(d \leq n)$ with discounted price processes $\left(S^{i}\right)_{i=1}^{d}=S$ modeled by

$$
d S_{t}=\operatorname{diag}\left(S_{t}\right)\left(b_{t} d t+\sigma_{t} d B_{t}\right), t \in[0, T], \mathcal{P}_{[\underline{a}, \bar{a}]} \text {-q.s., } \quad S_{0} \in(0, \infty)^{d},
$$

where $b$ (resp., $\sigma$ ) is a $\mathbb{R}^{d}$-valued (resp., $\mathbb{R}^{d \times n}$-valued) $\mathbb{F}$-predictable uniformly bounded process, with $\sigma$ being such that the family

$$
\left\{\int_{0}^{(P)} \sigma_{s} d B_{s}, P \in \mathcal{P}_{[\underline{a}, \bar{a}]}\right\} \text { aggregates into a single process } \int_{0}^{\cdot} \sigma_{s} d B_{s} .
$$

In addition, we assume that $\sigma \sigma^{\text {tr }}$ is uniformly elliptic in the sense that

$$
\text { there are } \Upsilon, \Lambda \in(0, \infty) \text { such that } \Upsilon \mathrm{I}_{d \times d} \leq \sigma \sigma^{\operatorname{tr}} \leq \Lambda \mathrm{I}_{d \times d}, \mathcal{P}_{[\underline{a}, \bar{a}]} \otimes d t \text {-q.e., }
$$

where $\mathrm{I}_{d \times d}$ denotes the $d \times d$ identity matrix. In particular, $\sigma \widehat{a}^{1 / 2}$ is $\mathcal{P}_{[\underline{a}, \bar{a}]} \otimes d t$-q.e. of maximal rank $d \leq n$, since $\sigma \widehat{a} \sigma^{\text {tr }}$ is uniformly elliptic and bounded (by (1),(8)).

Remark 6 For purposes of economic interpretation, it is desirable to have an aggregation of $S$, which should be quasi-surely defined as a single process. The latter is ensured here by the aggregation condition (7) which might seem restrictive at first sight, but is ensured, for instance, if $\sigma$ is càdlàg in which case $\int_{0}^{\cdot} \sigma_{s} d B_{s}$ can even be constructed pathwise as in Karandikar (1995).

The market model captures uncertainty about the volatility of the stock prices $S$ which is $\sigma \widehat{a}^{1 / 2}$ under each measure $P \in \mathcal{P}_{[\underline{a}, \bar{a}]}$. Since $d B_{t}=\widehat{a}_{t}^{1 / 2} d W_{t}^{P} P$-a.s. for a $P$-Brownian motion $W^{P}$, then the dynamics of $\left(S_{t}\right)_{t \in[0, T]}$ under $P \in \mathcal{P}_{[\underline{a}, \bar{a}]}$ is

$$
d S_{t}=\operatorname{diag}\left(S_{t}\right) \sigma_{t} \widehat{a}_{t}^{1 / 2}\left(\widehat{\xi}_{t} d t+d W_{t}^{P}\right), \text { with } \widehat{\xi}:=\widehat{a}^{1 / 2} \sigma^{\operatorname{tr}}\left(\sigma \widehat{a} \sigma^{\operatorname{tr}}\right)^{-1} b
$$

denoting the market price of risk in each reference model $P \in \mathcal{P}_{[\underline{a}, \bar{a}]}$. Note that $\widehat{\xi}$ is $\mathbb{R}^{n}$-valued, $\mathbb{F}$-predictable and uniformly bounded by a constant depending only on $\underline{a}, \bar{a}, \Lambda, \Upsilon$, and the uniform bound on $b$. The financial market described is thus typically incomplete for any reference measure $P \in \mathcal{P}_{[\underline{a}, \bar{a}]}$ for the volatility $\sigma \widehat{a}^{1 / 2}$ if $d<n$. In practice, the bounds $\underline{a}, \bar{a}$ and the uniform bounds on $\sigma \sigma^{\mathrm{tr}}$ can be viewed as describing some confidence region for future volatility values, which might be set, e.g., according to expert opinion about the range of historical or future (implied) volatility scenarios.

To also incorporate uncertainty about the drift, we admit for market prices of risk $\widehat{\xi}^{\theta}$ being from a radial set of which $\widehat{\xi}$ is the center, that is, we consider

$$
\left\{\widehat{\xi}^{\theta}:=\widehat{\xi}+\widehat{\Pi}(\theta) \mid \theta \mathbb{F} \text {-predictable, }\left|\theta_{t}(\omega)\right| \leq \delta_{t}(\omega),(t, \omega) \in[0, T] \times \boldsymbol{\Omega}\right\}
$$

where $\widehat{\Pi}_{(t, \omega)}(z):=\left(\sigma_{t}(\omega) \widehat{a}_{t}^{1 / 2}(\omega)\right)^{\operatorname{tr}}\left(\sigma_{t}(\omega) \widehat{a}_{t}(\omega) \sigma_{t}^{\operatorname{tr}}(\omega)\right)^{-1}\left(\sigma_{t}(\omega) \widehat{a}_{t}^{1 / 2}(\omega)\right) z$ denotes the orthogonal projection of $z \in \mathbb{R}^{n}$ onto $\operatorname{Im}\left(\widehat{a}_{t}^{1 / 2}(\omega) \sigma_{t}^{\operatorname{tr}}(\omega)\right), t \in[0, T]$, and $\delta$ is a fixed non-negative bounded $\mathbb{F}$-predictable process. The set (10) of market prices of 
risk corresponds to an ellipsoidal confidence region of drift uncertainty on risky asset prices $S$, such that ambiguous drifts could attain values in ellipsoids

$$
\left\{x \in \mathbb{R}^{d}:\left(x-b_{t}(\omega)\right)^{\operatorname{tr}}\left(\sigma_{t}(\omega) \widehat{a}_{t}(\omega) \sigma_{t}^{\operatorname{tr}}(\omega)\right)^{-1}\left(x-b_{t}(\omega)\right) \leq \delta_{t}^{2}(\omega)\right\},
$$

at $(t, \omega) \in[0, T] \times \boldsymbol{\Omega}$. Ellipsoidal specifications of uncertainty are common and appear naturally in the context of uncertainty about drift parameters in multivariate Gaussian settings, cf. e.g., Garlappi et al. (2007) or Biagini and Pinar (2017).

We denote by $\boldsymbol{\Theta}:[0, T] \times \boldsymbol{\Omega} \rightsquigarrow \mathbb{R}^{n}$ the correspondence (set-valued mapping)

$$
\boldsymbol{\Theta}_{t}(\omega):=\left\{x \in \mathbb{R}^{n}:|x| \leq \delta_{t}(\omega)\right\}, \quad \text { for }(t, \omega) \in[0, T] \times \boldsymbol{\Omega}
$$

with radial values. The notation “ $\rightsquigarrow$ ” emphasizes that $\boldsymbol{\Theta}$ is set-valued. $\mathbb{F}$-predictability of $\delta$ implies that $\boldsymbol{\Theta}$ is $\mathbb{F}$-predictable in the sense of Rockafellar (1976), i.e., the set $\boldsymbol{\Theta}^{-1}(F):=\left\{(t, \omega) \in[0, T] \times \boldsymbol{\Omega}: \boldsymbol{\Theta}_{t}(\omega) \cap F \neq \emptyset\right\}$ is $\mathbb{F}$-predictable for any closed set $F \subset \mathbb{R}^{n}$. Hence, by measurable selection arguments (cf. Rockafellar 1976, Cor.1.Q), $\boldsymbol{\Theta}$ admits $\mathbb{F}$-predictable selections, i.e., $\mathbb{F}$-predictable functions $\theta$ satisfying $\theta_{t}(\omega) \in \boldsymbol{\Theta}_{t}(\omega)$ for all $[0, T] \times \boldsymbol{\Omega}$. For an arbitrary correspondence $\Gamma$ : $[0, T] \times \Omega \rightsquigarrow \mathbb{R}^{n}$, we will write $\lambda \in \Gamma$ as a shortcut to mean that $\lambda$ is a function which is an $\mathbb{F}$-predictable selection of $\Gamma$. Moreover we will say that $\lambda$ is selection of $\Gamma$ (not necessarily measurable) if $\lambda_{t}(\omega) \in \Gamma_{t}(\omega)$ for all $(t, \omega) \in[0, T] \times \boldsymbol{\Omega}$.

Combined Knightian uncertainty (i.e., ambiguity) about drift and volatility scenarios is then captured by a (typically non-dominated) set

$$
\mathcal{R}:=\left\{Q: Q \sim P, d Q={ }^{(P)} \mathcal{E}\left(\theta \cdot W^{P}\right) d P \text { for } \theta \in \Theta, P \in \mathcal{P}_{[\underline{a}, \bar{a}]}\right\}
$$

of candidate reference probability measures (priors), where ${ }^{(P)} \mathcal{E}(M):=$ $\exp \left(M-M_{0}-\frac{1}{2}\langle M\rangle^{P}\right)$ denotes the stochastic exponential of a local martingale $M$ under $P$. For any $Q \in \mathcal{R}$, there are $P^{Q} \in \mathcal{P}_{[\underline{a}, \bar{a}]}, \theta^{Q} \in \Theta$ such that the canonical process $B$ is a $Q$-semimartingale with $B=\int_{0}^{\cdot} \widehat{a}_{s}^{1 / 2} \theta_{s}^{Q} d s+\int_{0}^{\cdot} \widehat{a}_{s}^{1 / 2} d W_{s}^{Q}$, where $W^{Q}=W^{P}-\int_{0}^{\cdot} \theta_{s}^{Q} d s$ is a $Q$-Brownian motion. We will simply denote by $Q^{P, \theta}$ a reference measure $Q \in \mathcal{R}$ associated to $P \in \mathcal{P}_{[\underline{a}, \bar{a}]}$ and $\theta \in \Theta$. Notice that the specification range for $P$ and $\theta$, respectively, accounts for uncertainty about volatilities and drifts. $S$ evolves under $Q^{P, \theta} \in \mathcal{R}, P$-a.s., as

$$
d S_{t}=\operatorname{diag}\left(S_{t}\right) \sigma_{t} \widehat{a}_{t}^{1 / 2}\left(\widehat{\xi}_{t}^{\theta} d t+d W_{t}^{P, \theta}\right),
$$

where $W^{P, \theta}:=W^{P}-\int_{0}^{\cdot} \theta_{s} d s$ is a $Q^{P, \theta}$-Brownian motion. Hence, $\widehat{\xi}^{\theta}$ is the market price of risk in the model $Q^{P, \theta}$, with volatility $\sigma \widehat{a}^{1 / 2}$ and $\widehat{a}$ satisfying $\underline{a} \leq \widehat{a} \leq$ $\bar{a}, P \otimes d t$-a.e.. Let $\mathcal{M}^{e}(Q):=\mathcal{M}^{e}(S, Q)$ denote the set of equivalent local martingale measures for $S$ in a model $Q$. Then, standard arguments (analogously to Becherer and Kentia 2017, Proposition 4.1) easily lead to the following

Lemma 2 For any $P \in \mathcal{P}_{[\underline{a}, \bar{a}]}$ and $\theta \in \boldsymbol{\Theta}$, the set $\mathcal{M}^{e}\left(Q^{P, \theta}\right)$ is equal to

$$
\begin{aligned}
& \left\{Q \sim Q^{P, \theta} \mid d Q={ }^{(P)} \mathcal{E}\left(\lambda \cdot W^{P, \theta}\right) d Q^{P, \theta}, \lambda=-\widehat{\xi}^{\theta}+\eta, \eta \in \operatorname{Ker}\left(\sigma \widehat{a}^{1 / 2}\right)\right\} \\
& =\left\{Q \sim P \mid d Q={ }^{(P)} \mathcal{E}\left(\lambda \cdot W^{P}\right) d P, \lambda=-\widehat{\xi}+\eta, \eta \in \operatorname{Ker}\left(\sigma \widehat{a}^{1 / 2}\right)\right\}=\mathcal{M}^{e}(P) .
\end{aligned}
$$


Remark 7 Note by Lemma 2 that for each $P \in \mathcal{P}_{[a, \bar{a}]}$ and $\theta \in \boldsymbol{\Theta}$, the minimal martingale measure $\widehat{Q}^{P, \theta}$ given by $d \widehat{Q}^{P, \theta}={ }^{(P)} \mathcal{E}\left(-\widehat{\xi}^{\theta} \cdot W^{P, \theta}\right) d Q^{P, \theta}$ is in $\mathcal{M}^{e}(P)$ since $\widehat{\xi}$ and $\delta$ are uniformly bounded. This implies that $\mathcal{M}^{e}(Q) \neq \emptyset$ for any $Q \in$ $\mathcal{R}$. Thus, the market satisfies the no-free lunch with vanishing risk condition (see Delbaen and Schachermayer 1994) under any (uncertain) prior $Q \in \mathcal{R}$. One can interpret this as a notion for no-arbitrage under drift and volatility uncertainty (as in, e.g., Biagini et al. 2017).

We parametrize trading strategies $\varphi=\left(\varphi^{i}\right)_{i=1}^{d}$ in terms of amount $\varphi^{i}$ of wealth invested in the stock with price process $S^{i}$, such that $\varphi$ is a $\mathbb{F}^{\mathcal{P}_{[a, a]} \text {-predictable process }}$ satisfying suitable integrability properties that will be made precise. In this respect, the wealth process $V^{\varphi}$ from a trading strategy $\varphi$ (in risky assets) that starts from some given initial capital $V_{0}$ has (for the self-financing requirement to be satisfied quasi-surely) the dynamics

$$
V_{t}^{\varphi}=V_{0}+\int_{0}^{t} \varphi_{s}^{\operatorname{tr}}\left(b_{s} d s+\sigma_{s} d B_{s}\right)=V_{0}+\int_{0}^{t} \varphi_{s}^{\operatorname{tr}} \sigma_{s}\left(\widehat{a}_{s}^{1 / 2} \widehat{\xi}_{s} d s+d B_{s}\right), \mathcal{P}_{[\underline{a}, \bar{a}]} \text {-q.s.. }
$$

on $[0, T]$. Re-parameterizing trading strategies in terms of integrands $\phi:=\sigma^{\operatorname{tr}} \varphi \in$ $\operatorname{Im} \sigma^{\text {tr }}$ w.r.t. $B+\int_{0}^{\cdot} \widehat{a}_{s}^{1 / 2} \widehat{\xi}_{s} d s$ yields as dynamics for the wealth process $V^{\phi}:=V^{\varphi}$

$$
V_{t}^{\phi}=V_{0}+\int_{0}^{t} \phi_{s}^{\mathrm{tr}}\left(\widehat{a}_{s}^{1 / 2} \widehat{\xi}_{s} d s+d B_{s}\right)=V_{0}+{ }^{(P)} \int_{0}^{t} \phi_{s}^{\mathrm{tr}} \widehat{a}_{s}^{\frac{1}{2}}\left(\widehat{\xi}_{s} d s+d W_{s}^{P}\right), t \in[0, T], P \text {-a.s. }
$$

for any $P \in \mathcal{P}_{[a, \bar{a}]}$. The set $\boldsymbol{\Phi}(P)$ of permitted strategies in the model $P$ (shortly $P$-permitted), $P \in \mathcal{P}_{[\underline{a}, \bar{a}]}$, is defined as

$$
\boldsymbol{\Phi}(P):=\left\{\phi \in \mathbb{H}^{2}\left(\mathbb{F}^{P}, P\right): \phi_{t}(\omega) \in \operatorname{Im} \sigma_{t}^{\mathrm{tr}}(\omega), \text { for all }(t, \omega) \in[0, T] \times \Omega\right\} .
$$

Under uncertainty and i.p. for non-dominated priors $P \in \mathcal{P}_{[\underline{a}, \bar{a}]}$, we want the wealth process for a trading strategy to be defined (q.e.) as single process, and not to vary with the prior. This requires as an additional condition on strategies $\phi$ that the family $\left\{{ }^{(P)} \int_{0}^{\cdot} \phi_{s}^{\mathrm{tr}} d B_{s}, P \in \mathcal{P}_{[\underline{a}, \bar{a}]}\right\}$ of "profit \& loss"-processes aggregates into a single process, denoted $\int_{0}^{\cdot} \phi_{s}^{\text {tr }} d B_{s}$. To this end, we make the

Definition 3 The set $\Phi$ of permitted trading strategies under drift and volatility uncertainty consists of all processes $\phi$ in $\mathbb{H}^{2}\left(\mathbb{F}^{\left.\mathcal{P}_{[\underline{a}, \bar{a}]}\right)}\right.$ with $\phi_{t}(\omega) \in \operatorname{Im} \sigma_{t}^{\text {tr }}(\omega)$ for all $(t, \omega) \in[0, T] \times \boldsymbol{\Omega}$, and being such that the family $\left\{{ }^{(P)} \int_{0}^{*} \phi_{s}^{t r} d B_{s}, P \in \mathcal{P}_{[\underline{a}, \bar{a}]}\right\}$ aggregates into a single process $\int_{0}^{*} \phi_{s}^{t r} d B_{s}$.

The trading strategies in $\boldsymbol{\Phi}$ will be termed as $\mathcal{P}_{[a, \bar{a}]}$-permitted (in short: permitted) strategies. By definition, one has $\boldsymbol{\Phi} \subseteq \boldsymbol{\Phi}(P)$ for all $P \in \mathcal{P}_{[\underline{a}, \bar{a}]}$. Hence, $V^{\phi}$ is a martingale under some measure equivalent to $P$ for any $\phi \in \boldsymbol{\Phi}$ and $P \in \mathcal{P}_{[\underline{a}, \bar{a}]}$. This excludes arbitrage strategies from $\boldsymbol{\Phi}$ for any scenario $\sigma \widehat{a}^{1 / 2}$ of the volatility.

Remark 8 a) Let us note, that we require the aggregation property in the definition above just because we want gains (i.e., profit \& loss) processes of strategies to 
be defined quasi-surely, for purposes of sensible economic interpretation in a setup with (non-dominated) uncertainty about P. But for the subsequent mathematical analysis, one could omit the assumption of aggregation - if one would be ready to consider strategies, whose gains processes cannot be defined quasi-surely, but where (P) $\int_{0}^{\cdot} \phi_{s}^{t r} d B_{s}$ could depend with $P \in \mathcal{P}_{[a, \bar{a}]}$. But we prefer not to.

b) By a result in Karandikar (1995), the aggregation property of Definition 3 would be satisfied, e.g., for $\phi$ being càdlàg and $\mathbb{F}$-adapted (like in Remark 6 for $\sigma$ ). Alternatively, aggregation would automatically hold under additional (non-standard) axioms for set-theory as in Nutz (2012a), cf. Remark 4.

\section{No-good-deal restriction and implications to dynamic valuation and hedging}

In the absence of uncertainty, we consider a (classical) no-good-deal restriction defined as a bound on the instantaneous Sharpe ratios, for any extension of the market by additional derivatives' prices computed by the no-good-deal pricing measures, following Björk and Slinko (2006), Cochrane and Saá-Requejo (2000). In a setup without jumps, such a constraint is equivalent to a bound on the optimal expected growth rate of returns for any market extension, cf. Becherer (2009). It is known that such is ensured by imposing a bound on the norm of the Girsanov kernels from the risk-neutral pricing measures. A slightly more detailed elaboration on the features of this valuation approach is given in Remark 9. Under solely drift uncertainty, robust good-deal hedging has been studied by Becherer and Kentia (2017). To extend the theory by including also ambiguity on volatility, we impose as no-good-deal restriction under combined drift and volatility uncertainty the same Sharpe ratio bound but require it under each model $Q \in \mathcal{R}$ separately. In doing so, we get for each $Q \in \mathcal{R}$ a set of no-good-deal measures $\mathcal{Q}^{\text {ngd }}(Q) \subseteq \mathcal{M}^{e}(Q)$. Following a worst-case approach to good-deal valuation under uncertainty, we then define the robust good-deal bound under drift and volatility uncertainty as the supremum of all no-good-deal bounds over all reference priors $Q \in \mathcal{R}$.

For clarity, we are going to explain the idea for the good-deal approach at first without addressing model uncertainty. To this end, let us assume, just for the remainder of this subsection, that we have neither ambiguity about drifts nor about volatility, and let us consider, just as a starting point, one arbitrary probability reference measure $Q^{P, \theta}$ from $\mathcal{R}$ to be the objective real world measure for the standard (non-robust) good deal problem of valuation and hedging. This means that we take one specification $P$ and $\theta$ for volatility and drift to be given. Having explained the problem without ambiguity, this will set the stage to explain the robust problem with model uncertainty thereafter in the main Section"Good-deal hedging and valuation under combined uncertainty".

\section{The no-good-deal problem under a given model $Q^{P, \theta}$ without uncertainty}

Let $h$ be a fixed non-negative bounded $\mathbb{F}$-predictable process. As prime example (see Cochrane and Saá-Requejo (2000), or the Section "A case study: Hedging a put option on a non-traded but correlated asset"), simply think of $h \geq 0$ to be a 
non-negative constant which defines a level beyond which Sharpe ratios are overly attractive, i.e., good deals. It can be understood as the parameter to specify preferences of investors, in order to move beyond the preference-free range of no-arbitrage reasoning, by using just "a little economics" (see Cochrane and Saá-Requejo 2000, commenting also on choice of $h$ and many applications). Admitting for dependence of $h$ on time and state $(t, \omega)$ is a mathematical generalization.

For a given probability measure $Q^{P, \theta}$ in $\mathcal{R}$ (with $P \in \mathcal{P}_{[\underline{a}, \bar{a}]}$ and $\theta \in \boldsymbol{\Theta}$ ), we consider the set $\mathcal{Q}^{\text {ngd }}\left(Q^{P, \theta}\right)$ of no-good-deal measures in the model $Q^{P, \theta}$ as the subset of $\mathcal{M}^{e}\left(Q^{P, \theta}\right)$ consisting of all equivalent local martingale measures $Q$, whose Girsanov kernels $\lambda$ w.r.t. the $Q^{P, \theta}$-Brownian motion $W^{P, \theta}$ are bounded by $h$ in the sense that $\left|\lambda_{t}(\omega)\right| \leq h_{t}(\omega)$ for all $(t, \omega) \in[0, T] \times \boldsymbol{\Omega}$. More precisely, using Lemma 2, we define

$$
\begin{gathered}
\mathcal{Q}^{\text {ngd }}\left(Q^{P, \theta}\right):=\left\{Q \sim Q^{P, \theta} \mid \frac{d Q}{d Q^{P, \theta}}={ }^{(P)} \mathcal{E}\left(\lambda \cdot W^{P, \theta}\right) \text {, for a } \mathbb{F}\right. \text {-predictable } \\
\left.\lambda=-\widehat{\xi}^{\theta}+\eta \text { with }|\lambda| \leq h \text { for } \eta \in \operatorname{Ker}\left(\sigma \widehat{a}^{\frac{1}{2}}\right)\right\} .
\end{gathered}
$$

In the sequel, we take $\boldsymbol{\Theta}$ to be such that any selection $\theta$ (not necessarily measurable) of $\boldsymbol{\Theta}$ satisfies

$$
\left|\widehat{\xi}_{t}^{\theta}(\omega)\right| \leq h_{t}(\omega), \text { for all }(t, \omega) \in[0, T] \times \boldsymbol{\Omega}, \theta \in \boldsymbol{\Theta},
$$

where $\widehat{\xi}^{\theta}$ is defined as in (10). Clearly, (15) implies that the minimal martingale measure $\widehat{Q}^{P, \theta}$ is in $\mathcal{Q}^{\text {ngd }}\left(Q^{P, \theta}\right) \neq \varnothing$ for any $P \in \mathcal{P}_{[\underline{a}, \bar{a}]}, \theta \in \boldsymbol{\Theta}$. Beyond this, (15) will also be used to prove wellposedness of a $2 \mathrm{BSDE}$ whose solution will describe the robust good-deal valuation bound and hedging strategy (see Theorems 1 and 2, respectively). As in (Becherer and Kentia 2017, Lemma 2.1, part b)), one can show for any $P \in \mathcal{P}_{[\underline{a}, \bar{a}]}$ and $\theta \in \Theta$ that the set $\mathcal{Q}^{\text {ngd }}\left(Q^{P, \theta}\right)$ is convex and multiplicatively stable (in short, $\mathrm{m}$-stable). The latter property, also referred to as rectangularity in the economic literature Chen and Epstein (2002), is usually required for time-consistency of essential suprema of conditional expectations over priors; see Delbaen (2006) for a general study.

For given $P \in \mathcal{P}_{[\underline{a}, \bar{a}]}$ and $\theta \in \boldsymbol{\Theta}$, the upper good-deal bound for a claim $X \in$ $L^{2}\left(\mathcal{F}_{T}^{P}, P\right)$ in model $Q^{P, \theta}$, with its given drift and volatility specification, is

$$
\pi_{t}^{u, P, \theta}(X):=\underset{Q \in \mathcal{Q}^{\text {ngd }}\left(Q^{P, \theta}\right)}{\operatorname{ess} \sup _{t}^{P}} E_{t}^{Q}[X], t \in[0, T], P \text {-a.s.. }
$$

Remark 9 According to classical good-deal theory in continuous time with continuous price dynamics, the set $\mathcal{Q}^{\text {ngd }}\left(Q^{P, \theta}\right)$ of risk-neutral measures $Q$ is specified in such a way, that in any extension $\left(S, S^{\prime}\right)$ of the financial market by additional derivatives' price processes $S^{\prime}$, which are (local) $Q$-martingales, the extended market is not just arbitrage-free but, moreover, does not permit opportunities for dynamic trading with overly attractive reward-to-risk ratios. More precisely, the choice of $\mathcal{Q}^{\text {ngd }}\left(Q^{P, \theta}\right)$ is such that the extended market does not offer trading opportunities that yield instantaneous Sharpe ratios (under probability measure $Q^{P, \theta}$ ) which exceed the bound $h$ 
(see Björk and Slinko 2006, Section 3 and Appendix A, for details); Equivalently, one can show that the extended market does not admit for portfolio strategies that offer (conditional) expected growth rates larger than h (see Becherer 2009, Section 3), and the respective good-deal restrictions are sharp. In this sense, the dynamic upper $\pi_{t}^{u, P, \theta}(X)$ and lower $-\pi_{t}^{u, P, \theta}(-X)$ good-deal bounds determine at any time $t$ a subinterval of the arbitrage-free prices, that is determined by those valuation measures $Q$ which restrict the opportunities for overly good deals.

The hedging objective for the seller of a claim $X \in L^{2}(P)$ who believes in the model $Q^{P, \theta}$ is to find a trading strategy $\bar{\phi}^{P, \theta} \in \boldsymbol{\Phi}(P)$ that minimizes the residual risk under a risk measure $\rho^{P, \theta}$ from holding $X$ and trading dynamically in the market. As the seller charges a premium $\pi^{u, P, \theta}(X)$ for $X$, she would like this premium to be the minimal capital that dynamically makes her position acceptable, so that $\pi^{u, P, \theta}$ becomes the market consistent risk measure corresponding to $\rho^{P, \theta}$, in the spirit of Barrieu and El Karoui (2009). As in Becherer (2009), we define $\rho^{P, \theta}$ as a dynamic coherent risk measure by

$$
\begin{aligned}
\rho_{t}^{P, \theta}(X) & :=\underset{Q \in \mathcal{P}^{\text {ngd }}\left(Q^{P, \theta}\right)}{\operatorname{ess} \operatorname{su}_{t}^{P}} E^{Q}[X], t \in[0, T], P \text {-a.s., } \quad \text { with } \\
\mathcal{P}^{\text {ngd }}\left(Q^{P, \theta}\right) & :=\left\{Q \sim P \mid \frac{d Q}{d Q^{P, \theta}}={ }^{(P)} \mathcal{E}\left(\lambda \cdot W^{P, \theta}\right), \lambda \mathbb{F} \text {-prog., }|\lambda| \leq h P \otimes d t \text {-a.e. }\right\} .
\end{aligned}
$$

Hence, $\mathcal{P}^{\text {ngd }}\left(Q^{P, \theta}\right)$ is the set of a-priori valuation measures equivalent to $Q^{P, \theta}$, which satisfy the no-good-deal restriction under $Q^{P, \theta}$ but might not be local martingale measures for the stock price process $S$ (yet they are w.r.t. the market with only the riskless asset $\left.S^{0} \equiv 1\right)$. This implies $\mathcal{Q}^{\text {ngd }}\left(Q^{P, \theta}\right)=\mathcal{P}^{\text {ngd }}\left(Q^{P, \theta}\right) \cap \mathcal{M}^{e}\left(Q^{P, \theta}\right)$, and thus $\rho_{t}^{P, \theta}(X) \geq \pi_{t}^{u, P, \theta}(X), t \in[0, T], P$-a.s. for all $X \in L^{2}(P)$. Moreover, as $\mathcal{Q}^{\text {ngd }}\left(Q^{P, \theta}\right)$ is $\mathrm{m}$-stable and convex, the set $\mathcal{P}^{\text {ngd }}\left(Q^{P, \theta}\right)$ also is, for any $P \in \mathcal{P}_{[\underline{a}, \bar{a}]}$ and $\theta \in \Theta$. Therefore, by (Becherer and Kentia 2017, Lemma 2.1) the dynamic (coherent) risk measure $\rho^{P, \theta}: L^{2}\left(\mathcal{F}_{T}^{P}, P\right) \rightarrow L^{2}\left(\mathcal{F}_{t}^{P}, P\right)$ is timeconsistent. For a fixed reference measure $Q^{P, \theta}$, the hedging problem for the seller of claim $X \in L^{2}\left(\mathcal{F}_{T}^{P}, P\right)$ is then to find $\bar{\phi}^{P, \theta} \in \Phi(P)$ such that $P$-almost surely for all $t \in[0, T]$ holds

$$
\begin{aligned}
\pi_{t}^{u, P, \theta}(X) & =\underset{\phi \in \Phi(P)}{\operatorname{essinf}} \rho_{t}^{P} \rho^{P, \theta}\left(X-{ }^{(P)} \int_{t}^{T} \phi_{s}^{\operatorname{tr}} \widehat{a}_{s}^{\frac{1}{2}}\left(\widehat{\xi}_{s} d s+d W_{s}^{P}\right)\right) \\
& =\rho_{t}^{P, \theta}\left(X-{ }^{(P)} \int_{t}^{T}\left(\bar{\phi}_{s}^{P, \theta}\right)^{\operatorname{tr}} \widehat{a}_{s}^{\frac{1}{2}}\left(\widehat{\xi}_{s} d s+d W_{s}^{P}\right)\right) .
\end{aligned}
$$

Remark 10 The approach to valuation and hedging-to-acceptability that shows in Eqs. (16)-(18) is close to the one from "conic finance", see, e.g., (Madan and Cherny 2010, Theorem 1) for analogous results in one period. Dynamic acceptability indices and performance measures are complementary concepts to dynamic (coherent) risk measures, for which good deal valuation bounds are a typical example (sign conventions aside). We refer to Bielecki et al. (2017) for a recent survey with many references to a rich literature, that delves into these connections and time-consistency 
problems, and to Bielecki et al. (2014) for a study of dynamic acceptability indices in discrete time. The results by Rosazza Gianin and Sgarra (2013) in continuous time build on BSDE techniques (i.e, "g-expectations") in a dominated setup, that appears closest to the one for our present Section "No-good-deal restriction and implications to dynamc valuation and hedging"; The analysis in their Section 3 shows, how a family of dynamic risk measures, which are indexed by a non-negative parameter, relates to an induced dynamic acceptability index. To make the link to their results, it suffices to consider our good deal bounds as being indexed (not by notation, but implicitly) by the non-negative parameter $h$, and to note that the bounds are described by the solutions to BSDEs (see Proposition 3) whose sublinear generators are monotone in $h$.

For a $P$-permitted trading strategy $\phi \in \Phi(P)$, we define the tracking (or hedging) error $R^{\phi}(X)$ of $\phi$ with respect to good-deal valuation $\pi^{u, P, \theta}(X)$ as

$$
R^{\phi}(X):=\pi^{u, P, \theta}(X)-\pi_{0}^{u, P, \theta}(X)-{ }^{(P)} \int_{0}^{\cdot} \phi_{s}^{\mathrm{tr}} \widehat{a}_{s}^{\frac{1}{2}}\left(\widehat{\xi}_{s} d s+d W_{s}^{P}\right),
$$

and it has (see Proposition 4 in Becherer and Kentia (2017)) the following property:

Proposition 2 For any $P$ in $\mathcal{P}_{[a, \bar{a}]}$ and $\theta$ in $\boldsymbol{\Theta}$, the tracking error $R^{\bar{\phi}^{P, \theta}}(X)$ of the good-deal hedging strategy $\bar{\phi}^{P, \theta}$ is a supermartingale under any measure $Q \in$ $\mathcal{P}^{n g d}\left(Q^{P, \theta}\right)$.

The supermartingale property of tracking errors as in Proposition 2 might already be viewed as a "robustness" property of the hedging strategy with respect to the family $\mathcal{P}^{\text {ngd }}\left(Q^{P, \theta}\right)$ of valuation probability measures as generalized scenarios (cf. Artzner et al. 1999). But we want to use the term robust only with respect to model uncertainty (ambiguity) about the real world probabilities, a situation to be explored in Section "Good-deal hedging and valuation under combined uncertainty", whereas in the present subsection the probability model $Q^{P, \theta}$ is assumed to be known, without uncertainty, for exposition purposes.

Standard BSDEs for valuation and hedging under $Q^{P, \theta}$ without uncertainty

The solution to the valuation and hedging problem described by (16) and (18) can be obtained in terms of standard BSDEs under $P \in \mathcal{P}_{[a, a]}$. In order to be more precise, let us introduce some notations that will also be used throughout the sequel. For $a \in$ $\mathbb{S}_{n}^{>0}$, we denote by $\Pi_{(t, \omega)}^{a}(\cdot)$ and $\Pi_{(t, \omega)}^{a, \perp}(\cdot)$, respectively, the orthogonal projections onto the subspaces $\operatorname{Im}\left(\sigma_{t}(\omega) a^{\frac{1}{2}}\right)^{\operatorname{tr}}$ and $\operatorname{Ker}\left(\sigma_{t}(\omega) a^{\frac{1}{2}}\right)$ of $\mathbb{R}^{n},(t, \omega) \in[0, T] \times \boldsymbol{\Omega}$. Explicitly, for each $a \in \mathbb{S}_{n}^{>0}$ and $t \in[0, T]$ (omitting $\omega$-symbols for simplicity), the projections for $z \in \mathbb{R}^{n}$ are given by

$$
\Pi_{t}^{a}(z)=\left(\sigma_{t} a^{1 / 2}\right)^{\mathrm{tr}}\left(\sigma_{t} a \sigma_{t}^{\mathrm{tr}}\right)^{-1}\left(\sigma_{t} a^{1 / 2}\right) z \quad \text { and } \quad \Pi_{t}^{a, \perp}(z)=z-\Pi_{t}^{a}(z) .
$$


In particular, we define $\widehat{\Pi}_{(t, \omega)}(\cdot):=\Pi_{(t, \omega)}^{\widehat{a}_{t}(\omega)}(\cdot)$ and $\widehat{\Pi}_{(t, \omega)}^{\perp}(\cdot):=\Pi_{(t, \omega)}^{\widehat{a}_{t}(\omega), \perp}(\cdot)$. For $P \in \mathcal{P}_{[\underline{a}, \bar{a}]}$ and $\theta \in \boldsymbol{\Theta}$, let us consider the standard BSDE under $P$,

$$
\mathcal{Y}_{t}=X-\int_{t}^{T} \widehat{F}_{s}^{\theta}\left(\mathcal{Y}_{s}, \widehat{a}_{s}^{1 / 2} \mathcal{Z}_{s}\right) d s-{ }^{(P)} \int_{t}^{T} \mathcal{Z}_{s}^{\mathrm{tr}} d B_{s}, t \in[0, T], P \text {-a.s. }
$$

for data $X \in L^{2}\left(\mathcal{F}_{T}^{P}, P\right)$ and generator $-\widehat{F}_{t}^{\theta}(\omega, \cdot)=-F^{\theta}\left(t, \omega \cdot \wedge t, \cdot, \widehat{a}_{t}(\omega)\right)$ with

$$
\begin{aligned}
F^{\theta}(t, \omega, z, a):= & -\Pi_{(t, \omega)}^{a, \perp}\left(\theta_{t}(\omega)\right)^{\operatorname{tr}} \Pi_{(t, \omega)}^{a, \perp}(z)+\widehat{\xi}_{t}^{\operatorname{tr}}(\omega) \Pi_{(t, \omega)}^{a}(z) \\
& -\left(h_{t}^{2}(\omega)-\left|\widehat{\xi}_{t}(\omega)+\Pi_{(t, \omega)}^{a}\left(\theta_{t}(\omega)\right)\right|^{2}\right)^{\frac{1}{2}}\left|\Pi_{(t, \omega)}^{a, \perp}(z)\right|
\end{aligned}
$$

for all $(t, \omega, z, a) \in[0, T] \times \boldsymbol{\Omega} \times \mathbb{R}^{n} \times \mathbb{S}_{n}^{>0}$. One can identify the upper valuation bound $\pi^{u, P, \theta}(X)$ and good-deal hedging strategy $\bar{\phi}^{P, \theta}$ in the model $Q^{P, \theta}$ in terms of solutions to the BSDE (19). More precisely, by (Becherer 2009, Theorem 5.4) holds

Proposition 3 For $P \in \mathcal{P}_{[a, \bar{a}]}$ and $\theta \in \boldsymbol{\Theta}$, let $X$ be a contingent claim in $L^{2}\left(\mathcal{F}_{T}^{P}, P\right)$ and $\left(\mathcal{Y}^{P, \theta}, \mathcal{Z}^{P, \theta}\right) \in \mathbb{D}^{2}\left(\mathbb{F}^{P}, P\right) \times \mathbb{H}^{2}\left(\mathbb{F}^{P}, P\right)$ be the unique solution to the standard BSDE (19). Then the good-deal bound $\pi^{u, P, \theta}(X)$ and the hedging strategy $\bar{\phi}^{P, \theta}$ in the model $Q^{P, \theta}$ are given by $\pi^{u, P, \theta}(X)=\mathcal{Y}^{P, \theta}$ and

$$
\widehat{a}_{t}^{\frac{1}{2}} \bar{\phi}_{t}^{P, \theta}=\widehat{\Pi}_{t}\left(\widehat{a}_{t}^{\frac{1}{2}} \mathcal{Z}_{t}^{P, \theta}\right)+\frac{\left|\Pi_{t}^{\perp}\left(\widehat{a}_{t}^{\frac{1}{2}} \mathcal{Z}_{t}^{P, \theta}\right)\right|}{\sqrt{h_{t}^{2}-\left|\widehat{\xi}_{t}+\widehat{\Pi}_{t}\left(\theta_{t}\right)\right|^{2}}}\left(\widehat{\xi}_{t}+\widehat{\Pi}_{t}\left(\theta_{t}\right)\right) \quad P \otimes d t \text {-a.e. } .
$$

The above standard BSDE description of good-deal bounds and hedging strategies has been extended by Becherer and Kentia (2017) to the presence of uncertainty solely about the drift, by using that drift uncertainty can be incorporated in a setup with one dominating reference measure. Yet, under volatility uncertainty, a dominating probability measure would fail to exist and hence standard BSDE techniques will not be applicable anymore. Instead, we will rely on 2BSDE theory to provide in Section "Good-deal hedging and valuation under combined uncertainty", under combined uncertainty about drifts and volatilities, a differential characterization of (robust) good-deal bounds and hedging strategies, after suitably defining the latter in such a typically non-dominated setup.

\section{Good-deal hedging and valuation under combined uncertainty}

We describe good-deal bounds in the market model of Section "Financial marketing with combined uncertainty about drift and volatility" using 2BSDEs and study a corresponding notion of hedging that is robust w.r.t. combined drift and volatility uncertainty. We first define the good-deal valuation bounds from the no-good-deal restriction of Section "No-good-deal restriction and implications to dynamic valua- 
tion and hedging", but take into account the investor's aversion towards drift and volatility uncertainty. Furthermore, still as in the Section "No-good-deal restriction and implications to dynamic valuation and hedging", hedging strategies are defined as minimizers of some dynamic coherent risk measure $\rho$ under uncertainty, so that the good-deal bound arises as the market consistent risk measure for $\rho$, in the spirit of Barrieu and El Karoui (2009), allowing for optimal risk sharing with the market under uncertainty.

For valuation, it seems natural to view uncertainty aversion as a penalization to the no-good-deal restriction in the sense that it implies good-deal bounds under uncertainty that are wider than in the absence of uncertainty. To formalize this idea, we rely on a classical worst-case approach to uncertainty in the spirit of, e.g., Gilboa and Schmeidler (1989). The idea is that an agent who is averse towards ambiguity about the actual drifts and volatilities may opt, to be conservative, for a worst-case approach to valuation in order to compensate for losses that could occur due to the wrong choice of model parameters. Acting this way, she would sell (resp., buy) financial risks at the largest upper (resp., smallest lower) good-deal bounds over all plausible priors in her confidence set $\mathcal{R}$ capturing drift and volatility uncertainty. Given the technical difficulties that may arise from $\mathcal{R}$ being non-dominated, in particular for writing essential suprema, we define the worst-case upper good-deal bound $\pi^{u}(X)$ for a financial risk $X \in L_{\mathcal{P}_{[\underline{a}, \bar{a}]}}^{2}$ as the unique process $\pi^{u}(X) \in \mathbb{D}^{2}\left(\mathbb{F}^{\mathcal{P}_{[\underline{a}, \bar{a}]}}\right)$ (if it exists) that satisfies

$$
\pi_{t}^{u}(X)=\operatorname{ess}_{P^{\prime} \in \mathcal{P}_{[\underline{a}, \bar{a}]}\left(t, P, \mathbb{F}_{+}\right)}^{P} \underset{\theta \in \Theta}{\operatorname{ess} \sup ^{P}} \pi_{t}^{u, P^{\prime}, \theta}(X), \quad t \leq T, P \text {-a.s., } P \in \mathcal{P}_{[\underline{a}, \bar{a}]},
$$

with $\pi^{u} . P^{\prime}, \theta(X)$ defined in (16). The lower bound $\pi^{l}(X)=-\pi^{u}(-X)$ is defined analogously, replacing essential suprema in (21) by essential infima, and for this reason we focus only on studying the upper bound. For $X \in \mathbb{L}^{2}\left(\mathbb{F}_{+}\right)$, we show in Section "Good-deal valuation bounds" that (21) indeed defines a single process $\pi^{u}(X)$ that can be identified as the $Y$-component of the solution of a specific 2BSDE.

For robust good-deal hedging, we define the hedging strategy similarly as in Section "No-good-deal restriction and implications to dynamic valuation and hedging", while taking into account model uncertainty. Indeed, under combined drift and volatility uncertainty, the hedging objective of the investor for a liability $X$ is to find a $\mathcal{P}_{[a, \bar{a}]}$-permitted trading strategy that dynamically minimizes the residual risk (under a suitable worst-case risk measure $\rho$ ) from holding the liability of the contingent claim $X$ and trading in the market. As a seller charging the premium $\pi^{u}(X)$ for $X$, she would like the upper good-deal bound to be the minimal capital to make her position $\rho$-acceptable at all times so that $\pi^{u}(\cdot)$ becomes the market consistent risk measure corresponding to $\rho .(\cdot)$. The second objective of the investor being robustness (of hedges and valuations) w.r.t. ambiguity about both drifts and volatilities, $\rho$ should be compatible with the no-good-deal restriction in the market and should also capture the investor's aversion towards uncertainty. From the definition of $\pi^{u}(X)$ (for $\left.X \in \mathbb{L}^{2}\left(\mathbb{F}_{+}\right)\right)$in (21) and the hedging problem (18) in the absence of uncertainty, we 
define $\rho .(X)$ for $X \in L_{\mathcal{P}_{[\underline{a}, \bar{a}]}}^{2}$ as the unique process in $\mathbb{D}^{2}\left(\mathbb{F}^{\mathcal{P}_{[\underline{a}, \bar{a}]}}\right)$ (if it exists) that satisfies, for $t \in[0, T]$,

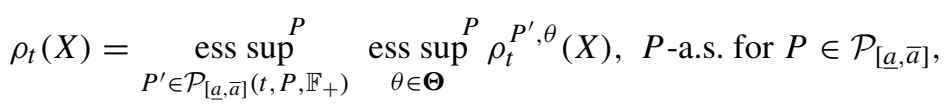

for $\rho^{P, \theta}(\cdot)$ defined in (17). The above considerations then lead to a good-deal hedging problem that is analogous to (18) in the case without uncertainty and, mathematically, for a $\mathcal{F}_{T}$-measurable contingent claim $X \in \mathbb{L}^{2}\left(\mathbb{F}_{+}\right)$writes: Find $\bar{\phi} \in \Phi$ such that for all $P \in \mathcal{P}_{[\underline{a}, \bar{a}]}$ holds $P$-almost surely for all $t \in[0, T]$,

$$
\begin{aligned}
\pi_{t}^{u}(X) & =\underset{\phi \in \Phi}{\operatorname{essinf}} P \rho_{t}\left(X-\int_{t}^{T} \phi_{s}^{\mathrm{tr}}\left(\widehat{a}_{s}^{1 / 2} \widehat{\xi}_{s} d s+d B_{s}\right)\right) \\
& =\rho_{t}\left(X-\int_{t}^{T} \bar{\phi}_{s}^{\mathrm{tr}}\left(\widehat{a}_{s}^{1 / 2} \widehat{\xi}_{s} d s+d B_{s}\right)\right) .
\end{aligned}
$$

Analogously to Becherer and Kentia (2017), we relate robustness of the hedging strategy (w.r.t. uncertainty) to a supermartingale property of its tracking error under a class $\mathcal{P}^{\text {ngd }}$ of a-priori valuation measures containing all $\mathcal{P}^{\text {ngd }}(Q)$, uniformly over all reference models $Q \in \mathcal{R}$. To introduce in more precise terms the notion of robustness w.r.t. ambiguity about drifts and volatilities, we define the tracking error $R^{\phi}(X)$ of a strategy $\phi \in \Phi$ for a claim $X \in \mathbb{L}^{2}\left(\mathbb{F}_{+}\right)$as

$$
R^{\phi}(X):=\pi_{.}^{u}(X)-\pi_{0}^{u}(X)-\int_{0}^{\cdot} \phi_{s}^{\operatorname{tr}}\left(\widehat{a}_{s}^{1 / 2} \widehat{\xi}_{s} d s+d B_{s}\right) .
$$

In words, $R_{t}^{\phi}(X)$ is the difference between the dynamic variations in the (monetary) capital requirement for $X$ and the profit $\&$ loss from trading (hedging) according to $\phi$ up to time $t$. Note that also $\pi^{u}$ is a dynamic coherent risk measure. Subsequently, we will say that a good-deal hedging strategy $\bar{\phi}(X)$ for a claim $X$ is robust w.r.t. (drift and volatility) uncertainty if $R^{\bar{\phi}}(X)$ is a $\left(\mathbb{F}^{P}, Q\right)$-supermartingale for every $Q \in \mathcal{P}^{\text {ngd }}\left(Q^{P, \theta}\right)$, uniformly for all $P \in \mathcal{P}_{[\underline{a}, \bar{a}]}$ and $\theta \in \Theta$.

Remark 11 If the tracking error $R^{\bar{\phi}}(X)$ is a $\left(\mathbb{F}^{P}, Q\right)$-supermartingale for any $Q$ in $\mathcal{P}^{n g d}\left(Q^{P, \theta}\right)$, then the strategy $\bar{\phi}$ is said to be "at least mean-self-financing" in the model $Q^{P, \theta}$, analogously to the property of being mean-self-financing (like riskminimizing strategies studied in Schweizer 2001, Section 2, with valuations taken under the minimal martingale measure) which would correspond to a martingale property (under $Q^{P, \theta}$ ) of the tracking errors.

We show in Section "Robust good-deal hedging" that the robust good-deal hedging strategy $\bar{\phi}$ can be obtained in terms of the control process of a 2BSDE describing the good-deal valuation bound $\pi^{u}$, and that the hedging strategy is robust with respect to combined uncertainty. This strategy will be shown to be quite different from the almost-sure hedging (i.e., superreplicating) strategy in general, in Section "A case study: Hedging a put option on a non-traded but correlated asset". 


\section{Good-deal valuation bounds}

For each $P \in \mathcal{P}_{[\underline{a}, \bar{a}]}, t \in[0, T]$ and $P^{\prime} \in \mathcal{P}_{[\underline{a}, \bar{a}]}\left(t, P, \mathbb{F}_{+}\right)$, the worst-case good-deal bound under drift uncertainty in the model $P^{\prime}$ is defined for $X \in L^{2}\left(\mathcal{F}_{T}^{P^{\prime}}, P^{\prime}\right)$ by

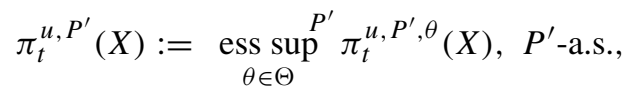

so that (21) rewrites for $\mathcal{F}_{T}$-measurable $X \in \mathbb{L}^{2}\left(\mathbb{F}_{+}\right)$as

$$
\pi_{t}^{u}(X)=\operatorname{ess}_{P^{\prime} \in \operatorname{Pup}_{[\underline{a}, \bar{a}]}\left(t, P, \mathbb{F}_{+}\right)}^{P} \pi_{t}^{u, P^{\prime}}(X), t \in[0, T], P \text {-a.s., } P \in \mathcal{P}_{[\underline{a}, \bar{a}]} .
$$

Note from (Becherer and Kentia 2017, Theorem 4.11) that the worst-case good-deal bound under drift uncertainty $\pi^{u, P}(X)$ for $P \in \mathcal{P}_{[\underline{a}, \bar{a}]}$ is the value process of the standard BSDE under $P$ with terminal condition $X$ and generator $-\widehat{F}(t, \omega, \cdot)=$ $-F\left(t, \omega \cdot \wedge t, \cdot, \widehat{a}_{t}(\omega)\right)$, where for all $(t, \omega, z, a) \in[0, T] \times \boldsymbol{\Omega} \times \mathbb{R}^{n} \times \mathbb{S}_{n}^{>0}$,

$$
F(t, \omega, z, a):=\inf _{\theta \in \mathbf{\Theta}} F^{\theta}(t, \omega, z, a)
$$

and $F^{\theta}$ is given by (20) for each selection $\theta$ of $\Theta$. With (25) and the above BSDE representation of $\pi^{u, P}(X), P \in \mathcal{P}_{[\underline{a}, \bar{a}]}$, the robust good-deal bound $\pi^{u}(X)$ can alternatively be formulated like in (Soner et al. 2013, Eq. (4.12) and Proposition 4.10) or, alternatively, (Possamaï et al. 2018, Eq. (2.6) and Lemma 3.5) as the value process of a stochastic control problem of nonlinear kernels. Roughly, this means to write, up to taking right-limits in time over rationals,

$$
\pi_{t}^{u}(X)(\omega)=\sup _{P \in \mathcal{P}_{[\underline{a}, \bar{a}]}^{t}} \pi_{t}^{u, P, t, \omega}(X) \quad \text { for all } \quad(t, \omega) \in[0, T] \times \Omega,
$$

where $\pi^{u, P, t, \omega}(X)$ are given by solutions to standard BSDEs under $P \in \mathcal{P}_{[\underline{a}, \bar{a}]}^{t}$, with terminal value $X$ and generator as in (26), but defined on the (shifted) canonical space $\boldsymbol{\Omega}^{t}:=\left\{\tilde{\omega} \in \mathcal{C}\left([t, T], \mathbb{R}^{n}\right): \tilde{\omega}(t)=0\right\}$ with (shifted) canonical process $B^{t}$, (shifted) natural filtration $\mathbb{F}^{t}$, and associated (shifted) set of priors $\mathcal{P}_{[\underline{a}, \bar{a}]}^{t}, t \in[0, T]$. Note that by a zero-one law as in Lemma $1, \pi_{t}^{u, P, t, \omega}(X)$ is indeed constant for any $(t, \omega)$ and $P \in \mathcal{P}_{[a, \bar{a}]}^{t}$ (like in Remark 12, Part 2.), and hence the pointwise supremum (27) is well-defined. Although such a pathwise description of the worst-case gooddeal bound $\pi_{t}^{u}(X)$ better reflects the economic intuition behind the latter and, as a stochastic control problem, would be more classical for the literature, it might be less suitable for approaching the hedging problem (23) for which the essential supremum formulation (25) seems more appropriate.

It is known (from Soner et al. 2012; Possamaï et al. 2018) that under suitable assumptions (on the terminal condition and the generator) the value process of a 
control problem like (27) can be described in terms of the solution to a $2 \mathrm{BSDE}$. We provide such a description for $\pi^{u}(X)$ by considering the 2BSDE

$$
Y_{t}=X-{ }^{(P)} \int_{t}^{T} Z_{s}^{\operatorname{tr}} d B_{s}-\int_{t}^{T} \widehat{F}_{s}\left(\widehat{a}_{s}^{1 / 2} Z_{s}\right) d s+K_{T}^{P}-K_{t}^{P}, t \in[0, T], \mathcal{P}_{[\underline{a}, \bar{a}]} \text {-q.s. }
$$

with generator $F$ given by (26) and terminal condition $X$. We have the following

Theorem 1 (Good-deal valuation under combined uncertainty) Let $X$ be $a$ $\mathcal{F}_{T}$-measurable claim in $\mathbb{L}^{2}\left(\mathbb{F}_{+}\right)$. Then the $2 B S D E$ (28) has a unique solution $\left(Y, Z,\left(K^{P}\right)_{P \in \mathcal{P}_{[\underline{a}, \bar{a}]}}\right) \in \mathbb{D}^{2}\left(\mathbb{F}^{\mathcal{P}_{[\underline{a}, \bar{a}]}}\right) \times \mathbb{H}^{2}\left(\mathbb{F}^{\mathcal{P}_{[\underline{a}, \bar{a}]}}\right) \times \mathbb{I}^{2}\left(\left(\mathbb{F}^{P}\right)_{P \in \mathcal{P}_{[a, \bar{a}]}}\right)$, the process $Y$ uniquely satisfies (21), and for any $P \in \mathcal{P}_{[\underline{a}, \bar{a}]}$ holds $\pi_{t}^{u}(X)=Y_{t}, t \in$ [0,T], P-a.s..

Proof We aim to apply Part 1. of Proposition 1 to show existence and uniqueness of the solution to the $2 \mathrm{BSDE}$ (28). To this end and since $X$ is $\mathcal{F}_{T}$-measurable and in $\mathbb{L}^{2}\left(\mathbb{F}_{+}\right)$, it suffices to check that $X$ and the function $F$ satisfy conditions (ii) to (iv) of Assumption 1. Because $\widehat{F}^{0} \equiv 0$, then (iv) obviously holds. As for (iii) about the uniform Lipschitz continuity of $F$ in $z$, it is enough to show that the functions $F^{\theta}$, for $\theta \in \Theta$, are equi-Lipschitz in $z$ uniformly for all $(t, \omega, a) \in[0, T] \times \boldsymbol{\Omega} \times \mathbb{S}_{n}^{>0}$. The latter holds since by Minkowski and Cauchy-Schwarz inequalities we get

$$
\begin{aligned}
&\left|F^{\theta}(t, \omega, z, a)-F^{\theta}\left(t, \omega, z^{\prime}, a\right)\right| \\
& \leq \mid\left|\Pi_{(t, \omega)}^{a, \perp}\left(\theta_{t}(\omega)\right)\right| \cdot\left|\Pi_{(t, \omega)}^{a, \perp}\left(z-z^{\prime}\right)\right|+\left|\widehat{\xi}_{t}(\omega)\right| \cdot\left|\Pi_{(t, \omega)}^{a}\left(z-z^{\prime}\right)\right| \\
& \quad+\left(h_{t}^{2}(\omega)-\left|\widehat{\xi}_{t}(\omega)+\Pi_{(t, \omega)}^{a}\left(\theta_{t}(\omega)\right)\right|^{2}\right)^{\frac{1}{2}}\left|\Pi_{(t, \omega)}^{a, \perp}\left(z-z^{\prime}\right)\right| \\
& \leq \delta_{t}(\omega)\left|z-z^{\prime}\right|+\left|\widehat{\xi}_{t}(\omega)\right|\left|z-z^{\prime}\right|+h_{t}(\omega)\left|z-z^{\prime}\right| \leq C\left|z-z^{\prime}\right|,
\end{aligned}
$$

for all $\theta \in \boldsymbol{\Theta}$, for $C \in(0, \infty)$, making use of (15) and boundedness of the functions $\delta$ and $h$. To infer the first claim of the theorem, it remains to show (ii) about $F$ being jointly and $\mathbb{F}$-progressively measurable. We first show that $F$ is $\mathbb{F}$-progressively measurable in $(t, \omega)$. For any $z \in \mathbb{R}^{n}, a \in \mathbb{S}_{n}^{>0}, t \in[0, T]$, one can rewrite $F^{\vartheta}(t, \cdot, z, a)$ from (20) as

$$
\begin{aligned}
& F^{\vartheta}(t, \cdot, z, a)=-\vartheta^{\operatorname{tr}} z+\vartheta^{\operatorname{tr}} a^{\frac{1}{2}} \sigma_{t}^{\operatorname{tr}}\left(\sigma_{t} a \sigma_{t}^{\operatorname{tr}}\right)^{-1} \sigma_{t} a^{\frac{1}{2}} z+\widehat{\xi}_{t}^{\operatorname{tr}} a^{\frac{1}{2}} \sigma_{t}^{\operatorname{tr}}\left(\sigma_{t} a \sigma_{t}^{\operatorname{tr}}\right)^{-1} \sigma_{t} a^{\frac{1}{2}} z \\
& -\left(h_{t}^{2}-\left|\widehat{\xi}_{t}+a^{\frac{1}{2}} \sigma_{t}^{\operatorname{tr}}\left(\sigma_{t} a \sigma_{t}^{\operatorname{tr}}\right)^{-1} \sigma_{t} a^{\frac{1}{2}} \vartheta\right|^{2}\right)^{\frac{1}{2}}\left(|z|^{2}-z^{\operatorname{tr}} a^{\frac{1}{2}} \sigma_{t}^{\operatorname{tr}}\left(\sigma_{t} a \sigma_{t}^{\operatorname{tr}}\right)^{-1} \sigma_{t} a^{\frac{1}{2}} z\right)^{\frac{1}{2}} .
\end{aligned}
$$

Since $\sigma, h, \widehat{\xi}$ are $\mathbb{F}$-predictable, the map $[0, T] \times \boldsymbol{\Omega} \times \mathbb{R}^{n} \ni(t, \omega, \vartheta) \mapsto F^{\vartheta}(t, \omega, z, a)$ is continuous in $\vartheta$ and $\mathbb{F}$-predictable in $(t, \omega)$. Moreover, as the correspondence $\Theta$ is $\mathbb{F}$-predictable, by measurable maximum and measurable selection results (Rockafellar 1976, Theorems $2 \mathrm{~K}, 1 . \mathrm{C})$ one has for each $(z, a) \in \mathbb{R}^{n} \times \mathbb{S}_{n}^{>0}$ that $F(\cdot, \cdot, z, a)$ is $\mathbb{F}$-predictable (hence $\mathbb{F}$-progressively measurable) and there exists $\theta^{z, a} \in \boldsymbol{\Theta}$ such that $F(t, \omega, z, a)=F^{\theta^{z, a}(t, \omega)}(t, \omega, z, a)=\inf _{\vartheta \in \Theta_{t}(\omega)} F^{\vartheta}(t, \omega, z, a)$ for all $(t, \omega) \in[0, T] \times \boldsymbol{\Omega}$. Now consider the correspondence $\widetilde{\boldsymbol{\Theta}}:[0, T] \times$ $\boldsymbol{\Omega} \times \mathbb{R}^{n} \times \mathbb{S}_{n}^{>0} \rightsquigarrow \mathbb{R}^{n}$ that is constant in its last two arguments and defined by 
$\widetilde{\boldsymbol{\Theta}}_{t}(\omega, z, a):=\boldsymbol{\Theta}_{t}(\omega)$ for all $(t, \omega, z, a) \in[0, T] \times \boldsymbol{\Omega} \times \mathbb{R}^{n} \times \mathbb{S}_{n}^{>0}$. Then $\widetilde{\boldsymbol{\Theta}}$ is a $\mathcal{P}(\mathbb{F}) \otimes \mathcal{B}\left(\mathbb{R}^{n}\right) \otimes \mathcal{B}\left(\mathbb{S}_{n}^{>0}\right)$-measurable correspondence because $\boldsymbol{\Theta}$ is $\mathbb{F}$-predictable, with $\mathcal{P}(\mathbb{F})$ denoting the predictable sigma-field w.r.t. $\mathbb{F}$. To prove joint measurability of $F$, it suffices by measurable selection arguments analogous to the ones above to show that the map $(t, \omega, z, a) \mapsto \inf _{\vartheta \in \widetilde{\Theta}_{t}(\omega, z, a)} F^{\vartheta}(t, \omega, z, a)$ is $\mathcal{B}([0, T]) \otimes \mathcal{F}_{T} \otimes$ $\mathcal{B}\left(\mathbb{R}^{n}\right) \otimes \mathcal{B}\left(\mathbb{S}_{n}^{>0}\right)$-measurable. Showing that $(t, \omega, z, a, \vartheta) \mapsto F^{\vartheta}(t, \omega, z, a)$ is a Carathéodory function would imply the result by (Rockafellar 1976, Theorem 2K). As $\vartheta \mapsto F^{\vartheta}(t, \omega, z, a)$ is continuous for each $(t, \omega, z, a)$, it remains only to show that $(t, \omega, z, a) \mapsto F^{\vartheta}(t, \omega, z, a)$ is jointly measurable for each $\vartheta$. First note that $F^{\vartheta}(\cdot, \cdot, z, a)$ is $\mathcal{B}([0, T]) \otimes \mathcal{F}_{T}$-measurable for each $(z, a, \vartheta) \in \mathbb{R}^{n} \times \mathbb{S}_{n}^{>0} \times \mathbb{R}^{n}$ since it is $\mathbb{F}$-predictable. Moreover, for any $x \in \mathbb{R}^{n \times k}, y \in \mathbb{R}^{n \times p}$ (with $k, p \in \mathbb{N}$ ) the function $\mathbb{R}^{n \times n} \ni a \mapsto x^{\text {tr }} a y \in \mathbb{R}^{k \times p}$ is continuous. Likewise, $a \mapsto a^{-1}$ and $a \mapsto a^{1 / 2}$ are continuous over $\mathbb{S}_{n}^{>0}$, the former being a consequence of the continuity of the determinant operator. Hence, from (29) one infers that the map $\mathbb{S}_{n}^{>0} \ni a \mapsto F^{\vartheta}(t, \omega, z, a)$ is continuous. Because $F^{\vartheta}$ is Lipschitz in $z$, uniformly in $(t, \omega, a)$, and continuous in $a$, it is continuous in $(z, a)$. As a Carathéodory function, $(t, \omega, z, a) \mapsto F^{\vartheta}(t, \omega, z, a)$ is jointly measurable and this concludes joint measurability of $F$. Overall, we have shown that $F$ satisfies Assumption 1. Hence the first claim of the theorem follows by Part 1. of Proposition 1 while the second claim is obtained from its Part 2., recalling the expression (25) for the good-deal bound and using (Becherer and Kentia 2017, Theorem 4.11).

Remark 12 1. In a situation with only volatility uncertainty and zero drift, one could apply, instead of Possamaï et al. (2018), an earlier but less general wellposedness result by Soner et al. (2012) to the 2BSDE (28), as noted in (Kentia 2015, [Theorem 4.20]). The latter requires (besides some continuity of $X$ ) the generator function $F$ to be convex (in a) and uniformly continuous (UC) in $\omega$, which would hold under UC-assumptions on $\sigma, h$. However, as soon as one considers non-zero drift (or even drift uncertainty), convexity of $F$ from our application is no longer clear. Uniform continuity for $F$ given by (26) in the present situation with combined uncertainties is neither. For example, a sufficient condition for it would be that the family $\left(F^{\theta}\right)_{\theta \in \Theta}$ is equicontinuous (in $\omega$ ); That, however, seems restrictive.

2. Theorem 1 shows, in particular, that the family of essential suprema in (21) indexed by the measures $P \in \mathcal{P}_{[\underline{a}, \bar{a}]}$ effectively aggregates into a single process $\pi^{u} \cdot(X) \in \mathbb{D}^{2}\left(\mathbb{F}^{\left[\mathcal{P}_{a}, \bar{a}\right]}\right)$ for Borel-measurable claims $X \in \mathbb{L}^{2}\left(\mathbb{F}_{+}\right)$. In this case, $\pi^{u}(X)$

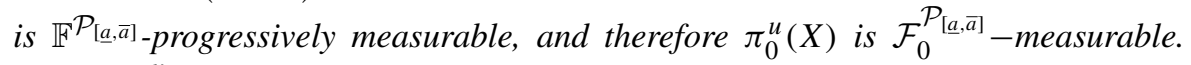
Hence, $\pi_{0}^{u}(X)$ is deterministic constant by the zero-one law of Lemma 1, with $\pi_{0}^{u}(X)=\sup _{P \in \mathcal{P}_{[a, \bar{a}]}} \pi_{0}^{u, P}(X)$.

3. By Proposition 1, the good-deal bound $\pi^{u}(\cdot)$ satisfies a dynamic programing principle for each Borel-measurable claim $X$ in $\mathbb{L}^{2}\left(\mathbb{F}_{+}\right)$: for any $P \in \mathcal{P}_{[\underline{a}, \bar{a}]}$ holds

$$
\pi_{s}^{u}(X)=\underset{P^{\prime} \in \mathcal{P}_{[a, a]}\left(s, P, \mathbb{F}_{+}\right)}{\operatorname{ess} \sup ^{P}} \pi_{s}^{u, P^{\prime}}\left(\pi_{t}^{u}(X)\right)=\pi_{s}^{u}\left(\pi_{t}^{u}(X)\right), s \leq t \leq T, P \text {-a.s. }
$$


4. Like in (Klöppel and Schweizer 2007, Theorem 2.7) (cf. (Becherer 2009, Proposition 2.6) or (Becherer and Kentia 2017, Lemma 2.1, Part a))) one can show that $(t, X) \mapsto \pi_{t}^{u}(X)$ has the properties of a time-consistent dynamic coherent risk measure.

\section{Robust good-deal hedging}

We now investigate robust good-deal hedging strategies relying on the 2BSDE theory of Section "Second-order backward stochastic differential equations". By (22), we have for $X \in L_{\mathcal{P}_{[\underline{a}, \bar{a}]}}^{2}$ that

$$
\begin{gathered}
\rho_{t}(X)=\underset{P^{\prime} \in \mathcal{P}_{[\underline{a}, \bar{a}]} \sup _{\left.t, P, \mathbb{F}_{+}\right)} P}{\operatorname{ess}} \rho_{t}^{P^{\prime}}(X), t \in[0, T], P \text {-a.s. for all } P \in \mathcal{P}_{[\underline{a}, \bar{a}]}, \\
\text { where } \rho_{t}^{P}(X):=\underset{\theta \in \Theta}{\operatorname{ess} \sup ^{P}} \rho_{t}^{P, \theta}(X), t \in[0, T] .
\end{gathered}
$$

As the set $\mathcal{P}^{\text {ngd }}\left(Q^{P, \theta}\right)$ is m-stable and convex for each $P \in \mathcal{P}_{[\underline{a}, \bar{a}]}, \theta \in \boldsymbol{\Theta}$, then by (Becherer and Kentia 2017, Lemma 4.9) the union $\bigcup_{\theta \in \Theta} \mathcal{P}^{\text {ngd }}\left(Q^{P, \theta}\right)$ is also $\mathrm{m}$-stable and convex. Thus the dynamic coherent risk measure $\rho^{P}: L^{2}(P) \rightarrow$ $L^{2}\left(P, \mathcal{F}_{t}\right)$ is time-consistent (see, e.g., Becherer and Kentia 2017, Lemma 2.1), with $\rho_{t}^{P}(X) \geq \pi_{t}^{u, P}(X), t \in[0, T], P$-a.s. for all $X \in L^{2}\left(P, \mathcal{F}_{T}\right)$. Consider the generator function $F^{\prime}:[0, T] \times \Omega \times \mathbb{R}^{n} \times \mathbb{S}_{n}^{>0} \rightarrow \mathbb{R}$ defined by

$$
F^{\prime}(t, \omega, z, a):=-\left(h_{t}+\delta_{t}\right)|z|, \quad \text { for all }(t, \omega, z, a) \in[0, T] \times \boldsymbol{\Omega} \times \mathbb{R}^{n} \times \mathbb{S}_{n}^{>0},
$$

and the associated 2BSDE

$$
Y_{t}^{\prime}=X-{ }^{(P)} \int_{t}^{T} Z_{s}^{\prime \operatorname{tr}} d B_{s}-\int_{t}^{T} \widehat{F}_{s}^{\prime}\left(\widehat{a}_{s}^{1 / 2} Z_{s}^{\prime}\right) d s+K_{T}^{\prime P}-K_{t}^{\prime P}, t \in[0, T], \mathcal{P}_{[\underline{a}, \bar{a}] \text {-q.s.. }}
$$

Proposition 4 below gives a 2BSDE description of $\rho .(X)$ that, similar to parts 3-4 of Remark 12 for $\pi^{u}(\cdot)$, implies that $\rho$ defines a dynamic risk measure (analogous to $\left.\pi^{u}(\cdot)\right)$ that is time-consistent over all $\mathcal{F}_{T}$-measurable $X$ in $\mathbb{L}^{2}\left(\mathbb{F}_{+}\right)$. The proof is analogous to that of Theorem 1, and its details are included in the Appendix.

Proposition 4 Let $X$ be a $\mathcal{F}_{T}$-measurable claim in $\mathbb{L}^{2}\left(\mathbb{F}_{+}\right)$. Then there exists a unique solution $\left(Y^{\prime}, Z^{\prime},\left(K^{\prime P}\right)_{\left.P \in \mathcal{P}_{[\underline{a}, \bar{a}]}\right)} \in \mathbb{D}^{2}\left(\mathbb{F}^{\mathcal{P}_{[\underline{a}, \bar{a}]}}\right) \times \mathbb{H}^{2}\left(\mathbb{F}^{\mathcal{P}_{[\underline{a}, \bar{a}]}}\right) \times\right.$ $\mathbb{I}^{2}\left(\left(\mathbb{F}^{P}\right)_{P \in \mathcal{P}_{[\underline{a}, \bar{a}]}}\right)$ to the $2 B S D E(32)$. Moreover, the process $Y^{\prime}$ uniquely satisfies (30) and for all $P \in \mathcal{P}_{[\underline{a}, \bar{a}]}$ holds $\rho_{t}(X)=Y_{t}^{\prime}, t \in[0, T], P$-a.s..

Note from results on good-deal valuation and hedging in the presence solely of drift uncertainty (cf. Becherer and Kentia 2017, Proposition 4.12, Theorem 4.13), that for any $P \in \mathcal{P}_{[\underline{a}, \bar{a}]}, P^{\prime} \in \mathcal{P}_{[\underline{a}, \bar{a}]}\left(t, P, \mathbb{F}_{+}\right)$and $X \in L^{2}\left(\mathcal{F}_{T}^{P}, P\right)$ one has P-a.s. that

$$
\pi_{t}^{u, P^{\prime}}(X)=\underset{\phi \in \Phi\left(P^{\prime}\right)}{\operatorname{essinf}} \rho_{t}^{P^{\prime}}\left(X-{ }^{\left(P^{\prime}\right)} \int_{t}^{T} \phi_{s}^{\mathrm{tr}}\left(\widehat{a}_{s}^{1 / 2} \widehat{\xi}_{s} d s+d B_{s}\right)\right), t \in[0, T] .
$$


Hence, from (25) and for $\mathcal{F}_{T}$-measurable claims $X$ in $\mathbb{L}^{2}\left(\mathbb{F}_{+}\right)$, it holds for all $P \in$ $\mathcal{P}_{[\underline{a}, \bar{a}]}, P$-almost surely, that for all $t \in[0, T]$.

$$
\pi_{t}^{u}(X)=\operatorname{ess}_{P^{\prime} \in \mathcal{P}_{[\underline{a}, \bar{a}]}\left(t, P, \mathbb{F}_{+}\right)}^{P} \underset{\phi \in \Phi\left(P^{\prime}\right)}{\operatorname{ess} \inf ^{P}} \rho_{t}^{P^{\prime}}\left(X-{ }^{\left(P^{\prime}\right)} \int_{t}^{T} \phi_{s}^{\mathrm{tr}}\left(\widehat{a}_{s}^{1 / 2} \widehat{\xi}_{s} d s+d B_{s}\right)\right),
$$

Furthermore, one can infer from (Becherer and Kentia 2017, Theorems 4.11 and 4.13) the existence of a family $\left\{\bar{\phi}^{P} \in \boldsymbol{\Phi}(P), P \in \mathcal{P}_{[\underline{a}, \bar{a}]}\right\}$ of trading strategies satisfying $P$-a.s. for all $t \in[0, T]$,

$$
\pi_{t}^{u}(X)=\operatorname{ess}_{P^{\prime} \in \mathcal{P}_{[\underline{a}, \bar{a}]}\left(t, P, \mathbb{F}_{+}\right)}^{P} \rho_{t}^{P^{\prime}}\left(X-{ }^{\left(P^{\prime}\right)} \int_{t}^{T}\left(\bar{\phi}_{s}^{P^{\prime}}\right)^{\operatorname{tr}}\left(\widehat{a}_{s}^{1 / 2} \widehat{\xi}_{s} d s+d B_{s}\right)\right)
$$

for each $P \in \mathcal{P}_{[\underline{a}, \bar{a}]}$. In addition, $\bar{\phi}^{P}$ is given for each $P \in \mathcal{P}_{[\underline{a}, \bar{a}]}$ by

$\widehat{a}_{t}^{\frac{1}{2}} \bar{\phi}_{t}^{P}=\widehat{\Pi}_{t}\left(\widehat{a}_{t}^{\frac{1}{2}} \mathcal{Z}_{t}^{P, X}\right)+\frac{\left|\Pi_{t}^{\perp}\left(\widehat{a}_{t}^{\frac{1}{2}} \mathcal{Z}_{t}^{P, X}\right)\right|}{\sqrt{h_{t}^{2}-\left|\widehat{\xi}_{t}+\widehat{\Pi}_{t}\left(\bar{\theta}_{t}^{P}\right)\right|^{2}}}\left(\widehat{\xi}_{t}+\widehat{\Pi}_{t}\left(\bar{\theta}_{t}^{P}\right)\right), P \otimes d t$-a.e.

where $\left(\mathcal{Y}^{P, X}, \mathcal{Z}^{P, X}\right)$ with $\mathcal{Y}^{P, X}=\pi^{u, P}(X)$ is the solution to the standard BSDE under $P \in \mathcal{P}_{[\underline{a}, \bar{a}]}$ with data $\left(-\widehat{F}\left(\widehat{a}^{\frac{1}{2}} \cdot\right), X\right)$ for $F$ defined in $(26)$, and $\bar{\theta}^{P}$ is an $\mathbb{F}^{P}$. predictable selection of $\boldsymbol{\Theta}$ satisfying

$$
\widehat{F}_{t}\left(\widehat{a}_{t}^{\frac{1}{2}} \mathcal{Z}_{t}^{P, X}\right)=\widehat{F}_{t}^{\bar{\theta}^{P}}\left(\widehat{a}_{t}^{\frac{1}{2}} \mathcal{Z}_{t}^{P, X}\right)=F^{\bar{\theta}^{P}}\left(t, \widehat{a}_{t}^{\frac{1}{2}} \mathcal{Z}_{t}^{P, X}, \widehat{a}_{t}\right) \quad P \otimes d t \text {-a.e. }
$$

with $F^{\bar{\theta}^{P}}$ given as in (20) for $\theta=\bar{\theta}^{P}$.

Remark 13 In the case of no volatility uncertainty (as studied in Becherer and Kentia 2017), i.e., for $\mathcal{P}_{[\underline{a}, \bar{a}]}=\left\{P^{0}\right\}$ with $\underline{a}=\bar{a}=I_{n \times n}$, the strategy $\bar{\phi}^{P^{0}}(X)$ for a claim $X$ in $L_{\mathcal{P}_{[\underline{a}, \bar{a}]}}^{2}\left(\mathcal{F}_{T}\right)=\mathbb{L}^{2}\left(\mathbb{F}_{+}\right)=L^{2}\left(\mathcal{F}_{T}, P^{0}\right)$ would be $\mathcal{P}_{[\underline{a}, \bar{a}] \text {-permitted and }}$ hence already the robust good-deal hedging strategy (w.r.t. drift uncertainty solely) for robust valuation $\pi^{u}(X)=\pi^{u}, P^{0}(X)$ and risk measure $\rho=\rho^{P^{0}}$. In the presence of volatility uncertainty however, the situation is more complicated because each strategy $\bar{\phi}^{P}$ and risk measure $\rho^{P}$ may be defined only up to a null-set of each (nondominated) measure $P \in \mathcal{P}_{[\underline{a}, \bar{a}]}$.

Since we are looking for a single process $\bar{\phi} \in \boldsymbol{\Phi}$ solution to the hedging problem (23), one way is to investigate appropriate conditions under which the family $\left\{\bar{\phi}^{P}, \quad P \in \mathcal{P}_{[\underline{a}, \bar{a}]}\right\}$ can be aggregated into a single strategy $\bar{\phi} \in \boldsymbol{\Phi}$, i.e., with 
$\bar{\phi}=\bar{\phi}^{P} P \otimes d t$-a.e., for all $P \in \mathcal{P}_{[\underline{a}, \bar{a}]}$. Were this possible, (34) would be written for any $P \in \mathcal{P}_{[a, \bar{a}]}$ as

$$
\begin{aligned}
\pi_{t}^{u}(X) & =\operatorname{ess}_{P^{\prime} \in \mathcal{P}_{[a, \bar{a}]}\left(t, P, \mathbb{F}_{+}\right)}^{P} \rho_{t}^{P^{\prime}}\left(X-\int_{t}^{T} \bar{\phi}_{s}^{\mathrm{tr}}\left(\widehat{a}_{s}^{1 / 2} \widehat{\xi}_{s} d s+d B_{s}\right)\right), t \in[0, T] . P \text {-a.s. } \\
& =\rho_{t}\left(X-\int_{t}^{T} \bar{\phi}_{s}^{\mathrm{tr}}\left(\widehat{a}_{s}^{1 / 2} \widehat{\xi}_{s} d s+d B_{s}\right)\right), t \in[0, T], P \text {-a.s., }
\end{aligned}
$$

such that the aggregate $\bar{\phi}$ would readily satisfy (23) since $\pi^{u}(\cdot) \leq \rho .(\cdot)$. However, since general conditions for aggregation are somewhat restrictive and highly technical (see, e.g., Soner et al. 2011), we shall abstain from following this path and will directly show that a suitable strategy described in terms of the $Z$-component of a 2BSDE (cf. (35) below) solves the good-deal hedging problem. In the simpler framework of drift uncertainty only, there exists a worst-case measure $\bar{P} \in \mathcal{R}$ such that $\pi_{\cdot}^{u}(\cdot)=\pi^{u,{ }_{P}}(\cdot)$ holds and the good-deal hedging strategy $\bar{\phi}^{\bar{P}}$ in the model $\bar{P}$ is robust w.r.t. (drift) uncertainty. This has been shown by first considering an auxiliary (larger) valuation bound for which the associated hedging strategy automatically satisfies the robustness property and then using a saddle point result (cf. Becherer and Kentia 2017, Theorem 4.13) to identify the auxiliary bound and hedging strategy with the standard good-deal bound and hedging strategy respectively. In the present nondominated framework, however, it appears questionable but indeed rather unlikely that such a worst-case measure $\bar{P}$ exists in $\mathcal{R}$ for general claims. Instead, we shall follow a slightly different approach and show in a more straightforward manner using Lemma 4 that the candidate strategy in (35) indeed satisfies the required robustness property w.r.t. drift and volatility uncertainty, which will then be used to recover its optimality. Note that our proof is not using a comparison theorem for 2BSDEs (as e.g. in Kentia 2015, Section 4.1.3). Such would need the terminal wealths $\int_{0}^{T} \phi_{s}^{\mathrm{tr}} d B_{s}$ as possible terminal conditions for $2 \mathrm{BSDEs}$ to be in $\mathbb{L}^{2}\left(\mathbb{F}_{+}\right)$, what might not be the case for $\phi \in \boldsymbol{\Phi}$.

For Borel-measurable $X$ in $\mathbb{L}^{2}\left(\mathbb{F}_{+}\right)$, consider the process $\bar{\phi}:=\bar{\phi}(X)$ defined by

$$
\widehat{a}_{t}^{\frac{1}{2}} \bar{\phi}_{t}:=\widehat{\Pi}_{t}\left(\widehat{a}_{t}^{\frac{1}{2}} Z_{t}\right)+\frac{\left|\Pi_{t}^{\perp}\left(\widehat{a}_{t}^{\frac{1}{2}} Z_{t}\right)\right|}{\sqrt{h_{t}^{2}-\left|\widehat{\xi}_{t}+\widehat{\Pi}_{t}\left(\bar{\theta}_{t}\right)\right|^{2}}}\left(\hat{\xi}_{t}+\widehat{\Pi}_{t}\left(\bar{\theta}_{t}\right)\right), \mathcal{P}_{[\underline{a}, \bar{a}]} \otimes d t \text {-q.e., }
$$

for the unique solution $\left(Y, Z,\left(K^{P}\right)_{P \in \mathcal{P}_{[a, \bar{a}]}}\right)$ to the $2 \operatorname{BSDE}(28), \bar{\theta}$ being a $\mathbb{F}^{\mathcal{P}_{[a, \bar{a}]_{-}}}$ predictable process with $\left|\bar{\theta}_{t}(\omega)\right| \leq \delta_{t}(\omega)$ for all $(t, \omega) \in[0, T] \times \boldsymbol{\Omega}$ and satisfying

$$
\widehat{F}_{t}\left(\widehat{a}_{t}^{\frac{1}{2}} Z_{t}\right)=\widehat{F}_{t}^{\bar{\theta}}\left(\widehat{a}_{t}^{\frac{1}{2}} Z_{t}\right)=F^{\bar{\theta}}\left(t, \widehat{a}_{t}^{\frac{1}{2}} Z_{t}, \widehat{a}_{t}\right), \mathcal{P}_{[\underline{a}, \bar{a}]} \otimes d t \text {-q.e.. }
$$

Existence of $\bar{\theta} \in \Theta$ satisfying (36) easily follows by measurable selection arguments similar to those in the proof of Theorem 1 , using the fact that $Z$ is $\mathbb{F}^{\mathcal{P}_{[a, \bar{a}]_{-}}}$ predictable, $\boldsymbol{\Theta}$ is $\mathbb{F}$-predictable and $\mathcal{P}(\mathbb{F}) \subseteq \mathcal{P}\left(\mathbb{F}^{\left[\frac{a}{a}, \bar{a}\right]}\right)$. The following result shows that $\bar{\phi}$ given by (35) is indeed a robust good-deal hedging strategy if the family $\left\{{ }^{(P)} \int_{0}^{\cdot} Z_{s}^{\mathrm{tr}} d B_{s}, P \in \mathcal{P}_{[\underline{a}, \bar{a}]}\right\}$ of "profit \& loss" processes aggregates. Note 
that, apart from measurability and some integrability, no regularity conditions (like, e.g. uniform continuity) are imposed on the contingent claim $X$. As the setup is non-Markovian, the contingent claim could clearly be path-dependent.

Theorem 2 (Robust good-deal hedging under combined uncertainty) For an $\mathcal{F}_{T}$-measurable $X$ in $\mathbb{L}^{2}\left(\mathbb{F}_{+}\right)$, let $\left(Y, Z,\left(K^{P}\right)_{P \in \mathcal{P}_{[\underline{a}, \bar{a}]}}\right)$ be the unique solution in

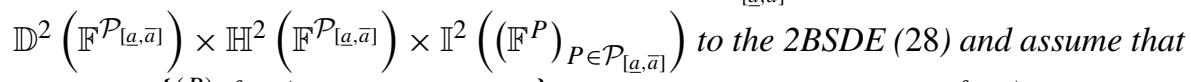
the family $\left\{{ }^{(P)} \int_{0}^{\cdot} Z_{s}^{t r} d B_{s}, P \in \mathcal{P}_{[\underline{a}, \bar{a}]}\right\}$ aggregates into one process $\int_{0}^{\cdot} Z_{s}^{\text {tr }} d B_{s}$. Then:

1. The process $\bar{\phi}=\bar{\phi}(X)$ from (35) is in $\boldsymbol{\Phi}$ and solves the good-deal hedging problem (23).

2. The tracking error $R^{\bar{\phi}}(X)$ (cf. (24)) of the good-deal hedging strategy $\bar{\phi}$ is a $\left(\mathbb{F}^{P}, Q\right)$-supermartingale under any $Q \in \mathcal{P}^{n g d}\left(Q^{P, \theta}\right)$, for all $P \in \mathcal{P}_{[\underline{a}, \bar{a}]}, \theta \in \mathbf{\Theta}$.

To prove Theorem 2, we will first show Part 2, and use it to prove Part 1. To this end, we will actually prove that the supermartingale property for a tracking error in (24) is sufficient for optimality of the corresponding strategy as in (23). Since optimality of a hedging strategy in turn clearly implies the supermartingale property for its tracking error, it follows that the latter property actually characterizes hedging strategies, as stated in the corollary that follows.

Corollary 1 For an $\mathcal{F}_{T}$-measurable $X$ in $\mathbb{L}^{2}\left(\mathbb{F}_{+}\right)$, a strategy $\bar{\phi}=\bar{\phi}(X) \in \boldsymbol{\Phi}$ solves the robust good-deal hedging problem (23) if and only if its tracking error $R^{\bar{\phi}}(X)$ is a $\left(\mathbb{F}^{P}, Q\right)$-supermartingale under any $Q \in \mathcal{P}^{n g d}\left(Q^{P, \theta}\right)$, for all $P \in$ $\mathcal{P}_{[\underline{a}, \bar{a}]}, \theta \in \Theta$.

We now provide the proof of Theorem 2 .

Proof (of Theorem 2) We first prove the second claim, and then use it to imply the first. Note that by the condition on the integrand $Z$, the strategy $\bar{\phi}$ given by (35) clearly belongs to $\boldsymbol{\Phi}$. By Theorem 1, we know that $\pi^{u}(X)=Y$ for $\left(Y, Z,\left(K^{P}\right)_{P \in \mathcal{P}_{[a, \bar{a}]}}\right)$ solution to the $2 \operatorname{BSDE}(28)$. Let $P \in \mathcal{P}_{[\underline{a}, \bar{a}]}, \theta \in \boldsymbol{\Theta}$ and $Q \in \mathcal{P}^{\text {ngd }}\left(Q^{P, \theta}\right)$. Then $Q$ is equivalent to $Q^{P, \theta}$ and $d Q={ }^{(P)} \mathcal{E}\left(\lambda \cdot W^{P, \theta}\right) d Q^{P, \theta}$ for $|\lambda| \leq h P \otimes d t$-a.e.. The dynamics of $R^{\bar{\phi}}:=R^{\bar{\phi}}(X)$ is then given $P$-almost surely for all $t \in[0, T]$ by

$$
\begin{aligned}
-d R_{t}^{\bar{\phi}} & =-\widehat{F}_{t}\left(\widehat{a}_{t}^{\frac{1}{2}} Z_{t}\right) d t-Z_{t}^{\operatorname{tr}} d B_{t}+\bar{\phi}_{t}^{\operatorname{tr}}\left(\widehat{a}_{t}^{\frac{1}{2}} \widehat{\xi}_{t} d t+d B_{t}\right)+d K_{t}^{P} \\
& =\left(\bar{\phi}_{t}^{\operatorname{tr}} \widehat{a}_{t}^{\frac{1}{2}} \widehat{\xi}_{t}-\widehat{F}_{t}\left(\widehat{a}_{t}^{\frac{1}{2}} Z_{t}\right)\right) d t-\left(Z_{t}-\bar{\phi}_{t}\right)^{\operatorname{tr}} \widehat{a}_{t}^{\frac{1}{2}} d W_{t}^{P}+d K_{t}^{P}
\end{aligned}
$$

By a change of measures from $P$ to $Q$ for the $Q$-Brownian motion $W^{Q}=W^{P}-$ $\int_{0}^{*}\left(\lambda_{t}+\theta_{t}\right) d t$, one obtains $P$-almost surely for all $t \in[0, T]$ that

$-d R_{t}^{\bar{\phi}}=\left(\bar{\phi}_{t}^{\operatorname{tr}} \widehat{a}_{t}^{\frac{1}{2}} \widehat{\xi}_{t}-\left(\lambda_{t}+\theta_{t}\right)^{\operatorname{tr}} \widehat{a}_{t}^{\frac{1}{2}}\left(Z_{t}-\bar{\phi}_{t}\right)-\widehat{F}_{t}\left(\widehat{a}_{t}^{\frac{1}{2}} Z_{t}\right)\right) d t-\left(Z_{t}-\bar{\phi}_{t}\right)^{\operatorname{tr}} \widehat{a}_{t}^{\frac{1}{2}} d W_{t}^{Q}+d K_{t}^{P}$. 
Since $\max |\lambda| \leq h \lambda_{t}^{\mathrm{tr}} \widehat{a}_{t}^{\frac{1}{2}}\left(Z_{t}-\bar{\phi}_{t}\right)=h_{t}\left|\widehat{a}_{t}^{\frac{1}{2}}\left(Z_{t}-\bar{\phi}_{t}\right)\right|, P \otimes d t$-a.e., then

$\bar{\phi}_{t}^{\mathrm{tr}} \widehat{a}_{t}^{\frac{1}{2}} \widehat{\xi}_{t}-\left(\lambda_{t}+\theta_{t}\right)^{\mathrm{tr}} \widehat{a}_{t}^{\frac{1}{2}}\left(Z_{t}-\bar{\phi}_{t}\right) \geq \bar{\phi}_{t}^{\mathrm{t}} \widehat{a}_{t}^{\frac{1}{2}} \widehat{\xi}_{t}-\theta_{t}^{\mathrm{t}} \widehat{a}_{t}^{\frac{1}{2}}\left(Z_{t}-\bar{\phi}_{t}\right)-h_{t}\left|\widehat{a}_{t}^{\frac{1}{2}}\left(Z_{t}-\bar{\phi}_{t}\right)\right|$,

$P \otimes d t$-a.e.. In addition, by Parts 1 and 2 of Lemma 4, one obtains from the definition (26) of $F$ and the expression (35) of $\bar{\phi}$ that $\widehat{F}_{t}\left(\widehat{a}_{t}^{\frac{1}{2}} Z_{t}\right)=\bar{\phi}_{t}^{\operatorname{tr}} \widehat{a}_{t}^{\frac{1}{2}} \widehat{\xi}_{t}-\bar{\theta}_{t}^{\operatorname{tr}} \widehat{a}_{t}^{\frac{1}{2}}\left(Z_{t}-\bar{\phi}_{t}\right)-$ $h_{t}\left|\widehat{a}_{t}^{\frac{1}{2}}\left(Z_{t}-\bar{\phi}_{t}\right)\right|, \mathcal{P}_{[\underline{a}, \bar{a}]} \otimes d t$-q.e., for $\bar{\theta} \in \boldsymbol{\Theta}$ satisfying (36). As a consequence, Part 3 of Lemma 4 yields

$$
\bar{\phi}_{t}^{\mathrm{tr}} \widehat{a}_{t}^{\frac{1}{2}} \widehat{\xi}_{t}-\theta_{t}^{\mathrm{tr}} \widehat{a}_{t}^{\frac{1}{2}}\left(Z_{t}-\bar{\phi}_{t}\right)-h_{t}\left|\widehat{a}_{t}^{\frac{1}{2}}\left(Z_{t}-\bar{\phi}_{t}\right)\right| \geq \widehat{F}_{t}\left(\widehat{a}_{t}^{\frac{1}{2}} Z_{t}\right), \mathcal{P}_{[\underline{a}, \bar{a}]} \otimes d t \text {-q.e.. }
$$

Hence, since $K^{P}$ is non-decreasing, then combining (37) and (38) imply that the finite variation part of the $Q$-semimartingale $R^{\bar{\phi}}$ is non-increasing. Furthermore, one has $R^{\bar{\phi}} \in \mathbb{D}^{2}\left(\mathbb{F}^{P}, P\right)$ because $\pi^{u}(X) \in \mathbb{D}^{2}\left(\mathbb{F}^{\mathcal{P}[a, \bar{a}]}\right) \subseteq \mathbb{D}^{2}\left(\mathbb{F}^{P}, P\right)$ and $\bar{\phi} \in \Phi \subseteq$ $\Phi(P)$. Now, since $\lambda+\theta$ is bounded, then $\left.\frac{d Q}{d P}\right|_{\mathcal{F}_{T}}$ is in $L^{p}\left(\mathcal{F}_{T}, P\right)$ for any $p<\infty$ and by Hölder's inequality it follows that $R^{\bar{\phi}} \in \mathbb{D}^{2-\epsilon}\left(\mathbb{F}^{P}, Q\right)$ holds for some $\epsilon>0$. Thus, $R^{\bar{\phi}}$ is a $\left(\mathbb{F}^{P}, Q\right)$-supermartingale.

We turn to the proof of the first claim of the theorem. To show that $\bar{\phi}$ solves problem (23), let $P \in \mathcal{P}_{[\underline{a}, \bar{a}]}$. By (33) and $\Phi \subseteq \Phi\left(P^{\prime}\right)$ for $P^{\prime} \in \mathcal{P}_{[\underline{a}, \bar{a}]}$, then $P$-a.s.

$$
\begin{aligned}
& \pi_{t}^{u}(X) \leq \operatorname{ess}_{P^{\prime} \in \mathcal{P}_{[a, \bar{a}]}\left(t, P, \mathbb{F}_{+}\right)}^{P} \underset{\phi \in \Phi}{\operatorname{essinf}} P_{t}^{P} \rho_{t}^{P^{\prime}}\left(X-\int_{t}^{T} \phi_{s}^{\mathrm{tr}}\left(\widehat{a}_{s}^{1 / 2} \widehat{\xi}_{s} d s+d B_{s}\right)\right) \\
& \leq \underset{\phi \in \Phi}{\operatorname{essinf}} \operatorname{Ps}_{\left.P^{\prime} \in \mathcal{P}_{[a, a]}\right]\left(t, P, \mathbb{F}_{+}\right)}^{P} \rho_{t}^{P^{\prime}}\left(X-\int_{t}^{T} \phi_{s}^{\operatorname{tr}}\left(\widehat{a}_{s}^{1 / 2} \widehat{\xi}_{s} d s+d B_{s}\right)\right) \\
& =\underset{\phi \in \Phi}{\operatorname{ess} \inf ^{P}} \rho_{t}\left(X-\int_{t}^{T} \phi_{s}^{\mathrm{tr}}\left(\widehat{a}_{s}^{1 / 2} \widehat{\xi}_{s} d s+d B_{s}\right)\right) \quad \text { for } t \in[0, T] .
\end{aligned}
$$

To conclude that some $\bar{\phi} \in \boldsymbol{\Phi}$ is a good-deal hedging strategy satisfying (23), it suffices to show for all $\theta \in \boldsymbol{\Theta}, P \in \mathcal{P}_{[\underline{a}, \bar{a}]}$ that $P$-a.s. for all $t \in[0, T], P^{\prime} \in$ $\mathcal{P}_{[\underline{a}, \bar{a}]}\left(t, P, \mathbb{F}_{+}\right)$and $Q \in \mathcal{P}^{\text {ngd }}\left(Q^{P^{\prime}, \theta}\right)$ holds

$$
\pi_{t}^{u}(X) \geq E_{t}^{Q}\left[X-\int_{t}^{T} \bar{\phi}_{s}^{\mathrm{tr}}\left(\widehat{a}_{s}^{1 / 2} \widehat{\xi}_{s} d s+d B_{s}\right)\right] .
$$

To this end, let $\theta \in \boldsymbol{\Theta}, P \in \mathcal{P}_{[a, \bar{a}]}$. By the second part of the theorem, the tracking error $R^{\bar{\phi}}:=R^{\bar{\phi}}(X)$ of $\bar{\phi}$ is a $\left(\mathbb{F}^{P^{\prime}}, Q\right)$-supermartingale for every $Q \in$ $\mathcal{P}^{\text {ngd }}\left(Q^{P^{\prime}, \theta}\right), P^{\prime} \in \mathcal{P}_{[\underline{a}, \bar{a}]}$. This implies by Lemma 1 that $\pi_{t}^{u}(X)-\pi_{0}^{u}(X)-$ $\int_{0}^{t} \bar{\phi}_{s}^{\text {tr }}\left(\widehat{a}_{s}^{1 / 2} \widehat{\xi}_{s} d s+d B_{s}\right) \geq E_{t}^{Q}\left[X-\pi_{0}^{u}(X)-\int_{0}^{T} \bar{\phi}_{s}^{\text {tr }}\left(\widehat{a}_{s}^{1 / 2} \widehat{\xi}_{s} d s+d B_{s}\right)\right], P$-a.s., for all $Q \in \mathcal{P}^{\text {ngd }}\left(Q^{P^{\prime}, \theta}\right), P^{\prime} \in \mathcal{P}_{[\underline{a}, \bar{a}]}\left(t, P, \mathbb{F}_{+}\right)$, for any $t \leq T$. Reorganizing that inequality yields the claim. 
Remark 14 a) Theorem 2 yields a minmax identity: For $P \in \mathcal{P}_{[\underline{a}, \bar{a}]}$ holds a.s.

$$
\begin{aligned}
\pi_{t}^{u}(X) & =\underset{P^{\prime} \in \mathcal{P}_{[\underline{a}, \bar{a}]}\left(t, P, \mathbb{F}_{+}\right)}{\operatorname{ess} \sup ^{P} \underset{\phi \in \boldsymbol{\Phi}\left(P^{\prime}\right)}{\operatorname{essinf}} P} \rho_{t}^{P^{\prime}}\left(X-{ }^{(P)} \int_{t}^{T} \phi_{s}^{t r}\left(\widehat{a}_{s}^{1 / 2} \widehat{\xi}_{s} d s+d B_{s}\right)\right) \\
& =\underset{\phi \in \Phi}{\operatorname{essinf} P} \underset{P^{\prime} \in \mathcal{P}_{[\underline{a}, \bar{a}]}\left(t, P, \mathbb{F}_{+}\right)}{P} \rho_{t}^{\operatorname{ess~sup}^{\prime}} \rho^{P^{\prime}}\left(X-\int_{t}^{T} \phi_{s}^{t r}\left(\widehat{a}_{s}^{1 / 2} \widehat{\xi}_{s} d s+d B_{s}\right)\right), \quad t \in[0, T] .
\end{aligned}
$$

b) By a result of Karandikar (1995), one can define the stochastic integral $\int_{0}^{\cdot} Z_{t}^{t r} d B_{t}$ pathwise if the integrand $Z$ is càdlàg and $\mathbb{F}$-adapted, such that, in particular, it satisfies the hypothesis of Theorem 2. See also Remark 8. Although the $Z$-component of a 2BSDE solution does not need to be càdlàg in general, let us note how Theorem 2 can still be applicable in some situations. For instance, in a Markovian diffusion setting, one may be able to use partial differential equation (PDE) arguments to show that the Z-component is even continuous. An example in such a setting is provided in Section "A case study: Hedging a put option on a non-traded but correlated asset”, where beyond the continuity of $Z$ we can even obtain explicit solutions to the $2 B S D E$ (28), for some bounded contingent claims. For a general situation, however, a result by Nutz (2012a) can be used under additional set-theoretical assumptions to get rid of the aggregation condition in $\Phi$ and in the statement of Theorem 2; cf. Remarks 4 and 8.

\section{A case study: Hedging a put option on a non-traded but correlated asset}

In this subsection, we investigate a simple Markovian example to provide more intuition and to illustrate the general but abstract main theorems for robust good-deal valuations and hedges, by giving some explicit closed-form formulas. To this end, we investigate the particular application of a vanilla put option on a non-traded asset in concrete detail. The latter is a typical problem for optimal partial hedging in incomplete markets. The example is elementary enough to permit even for closedform solutions, in some parameter settings, and can also be solved by more standard optimal control methods, exploiting the Markovian structure and certain properties of the claim's payoff function. We will make use of this fact to demonstrate clear differences to hedging by superreplication, and further to elucidate the difficulties arising from combined drift and volatility uncertainty, compared to only one type of ambiguity. More precisely, we show how under combined uncertainty the optimal control problem of robust good-deal valuation becomes effectively one over a non-rectangular domain of control variables, making it more complex to identify optimizers and worst-case priors (or parameters), even for monotone convex payoff functions for which intuition from examples in other related valuation approaches (e.g., robust superreplication) might at first suggest otherwise. Of course, one cannot expect to get similarly elementary solutions for measurable contingent claims in general models: For the general case, the solution is fully characterized by means of 2BSDEs in the main Theorems 1 and 2.

Let us consider a financial market where only one risky asset (a stock) with discounted price process $S$ is tradeable, apart from the riskless asset (with unit price). In addition, there is another asset that is not tradeable but whose value $L$ is correlated 
with $S$. The processes $S$ and $L$ are, $\mathcal{P}_{[\underline{a}, \bar{a}]}$-q.s., given by

$$
d S_{t}=S_{t}\left(b d t+\sigma^{S} d B_{t}^{1}\right) \quad \text { and } \quad d L_{t}=L_{t}\left(\gamma d t+\beta\left(\rho d B_{t}^{1}+\sqrt{1-\rho^{2}} d B_{t}^{2}\right)\right),
$$

where $B=\left(B^{1}, B^{2}\right)$ is the canonical process, $\mathcal{P}_{[\underline{a}, \bar{a}]}$ is the set defined as in (1) for diagonal matrices $\underline{a}=\operatorname{diag}\left(\underline{a}_{1}, \underline{a}_{2}\right)$ and $\bar{a}=\operatorname{diag}\left(\bar{a}_{1}, \bar{a}_{2}\right)$ in $\mathbb{S}_{2}^{>0}$, with $S_{0}, L_{0}, \sigma^{S}, \beta$ in $(0, \infty)$, for drifts $b, \gamma$ in $\mathbb{R}$, volatility matrix $\sigma:=\left(\sigma^{S}, 0\right) \in \mathbb{R}^{1 \times 2}$, and a $P^{0}$-correlation coefficient $\rho \in[-1,1]$. For a constant bound $h \in[0, \infty)$ on the instantaneous Sharpe ratios, we are going to derive closed-form expressions for robust good-deal valuations and hedges for a European put option $X:=\left(\mathcal{K}-L_{T}\right)^{+}$ on the non-traded asset $L$ with strike $\mathcal{K} \in(0, \infty)$ and maturity $T$, and to identify the corresponding worst-case drifts and volatilities. At first we assume the drift rate $b$ of $S$ to be zero, so that the center market price of risk $\widehat{\xi}$ vanishes quasi-surely, and consider the case of uncertainty solely on volatility (i.e., for $\delta \equiv 0$, that is $\boldsymbol{\Theta}=\{0\}$ in (11)). For this case we will identify a worst-case volatility for the robust valuation bounds in closed form, and compare robust good-deal hedging with classical robust superreplication under volatility uncertainty. The case with uncertainty solely about the drifts of tradeable assets (i.e. for $\underline{a}=\bar{a}=I_{n \times n}$ ) has been solved in (Becherer and Kentia 2017, Section 4.6). We proceed by discussing the more complex case with combined drift and volatility uncertainties and the difficulties that arise in identifying worst-case drifts and volatilities. Finally, we investigate sensitivities of the derived robust good-deal bound under volatility uncertainty with respect to variations of the drift parameter $\gamma \in \mathbb{R}$ for the non-traded asset.

\section{Uncertainty solely about the volatility}

Denoting the entries of the processes $\widehat{a}$ and its square root $\widehat{a}^{\frac{1}{2}}$ by

$$
\widehat{a}=\left(\begin{array}{ll}
\widehat{a}^{11} & \widehat{a}^{12} \\
\widehat{a}^{12} & \widehat{a}^{22}
\end{array}\right) \text { and } \widehat{a}^{\frac{1}{2}}=\left(\begin{array}{cc}
\widehat{c}^{11} & \widehat{c}^{12} \\
\widehat{c}^{12} & \widehat{c}^{22}
\end{array}\right),
$$

one has $\sigma \widehat{a}^{\frac{1}{2}}=\sigma^{S}\left(\widehat{c}^{11}, \widehat{c}^{12}\right)$, and furthermore the identities

$$
\begin{gathered}
\widehat{a}^{11}=\left(\widehat{c}^{11}\right)^{2}+\left(\widehat{c}^{12}\right)^{2}, \quad \widehat{a}^{12}=\widehat{c}^{12}\left(\widehat{c}^{11}+\widehat{c}^{22}\right), \quad \widehat{a}^{22}=\left(\widehat{c}^{22}\right)^{2}+\left(\widehat{c}^{2}\right)^{2}, \\
\text { and } \widehat{a}^{11} \widehat{a}^{22}-\left(\widehat{a}^{12}\right)^{2}=\left(\widehat{c}^{11} \widehat{c}^{22}-\left(\widehat{c}^{12}\right)^{2}\right)^{2},
\end{gathered}
$$

implying $\operatorname{Im}\left(\sigma \widehat{a}^{\frac{1}{2}}\right)^{\operatorname{tr}}=\left\{z \in \mathbb{R}^{2}: \widehat{c}^{12} z_{1}-\widehat{c}^{11} z_{2}=0\right\}$ and $\operatorname{Ker}\left(\sigma \widehat{a}^{\frac{1}{2}}\right)=$ $\left\{z \in \mathbb{R}^{2}: \widehat{c}^{11} z_{1}+\widehat{c}^{12} z_{2}=0\right\}$. Hence, for $z=\left(z_{1}, z_{2}\right)^{\text {tr }} \in \mathbb{R}^{2}$ one gets

$$
\widehat{\boldsymbol{\Pi}}(z)=\frac{1}{\widehat{a}^{11}}\left(\begin{array}{c}
\left(\widehat{c}^{11}\right)^{2} z_{1}+\widehat{c}^{11} \widehat{c}^{12} z_{2} \\
\widehat{c}^{11} \widehat{c}^{12} z_{1}+\left(\widehat{c}^{12}\right)^{2} z_{2}
\end{array}\right) \text { and } \widehat{\Pi}^{\perp}(z)=\frac{1}{\widehat{a}^{11}}\left(\begin{array}{c}
\left(\widehat{c}^{12}\right)^{2} z_{1}-\widehat{c}^{11} \widehat{c}^{12} z_{2} \\
\left(\widehat{c}^{11}\right)^{2} z_{2}-\widehat{c}^{11} \widehat{c}^{12} z_{1}
\end{array}\right) .
$$

In the case $\delta \equiv 0$ (no drift uncertainty), the $2 \mathrm{BSDE}$ (28) rewrites here as

$$
Y_{t}=X-{ }^{(P)} \int_{t}^{T} Z_{s}^{\mathrm{tr}} d B_{s}-\int_{t}^{T} \widehat{F}_{s}\left(\widehat{a}_{s}^{1 / 2} Z_{s}\right) d s+K_{T}^{P}-K_{t}^{P}, t \in[0, T], \mathcal{P}_{[\underline{a}, \bar{a}] \text {-q.s. },}
$$


where from (40) and (39) one has $\mathcal{P}_{[\underline{a}, \bar{a}]} \otimes d t$-q.e.

$$
\widehat{F}_{t}\left(\widehat{a}_{t}^{1 / 2} z\right)=-h\left|\widehat{\boldsymbol{\Pi}}_{t}^{\perp}\left(\widehat{a}_{t}^{1 / 2} z\right)\right|=-h\left(\widehat{a}_{t}^{11} \widehat{a}_{t}^{22}-\left(\widehat{a}_{t}^{12}\right)^{2}\right)^{1 / 2}\left(\widehat{a}_{t}^{11}\right)^{-1 / 2}\left|z_{2}\right|,
$$

for $z=\left(z_{1}, z_{2}\right)^{\operatorname{tr}} \in \mathbb{R}^{2}$. Clearly, $L_{T}$ is $\mathcal{F}_{T}$-measurable, and since the put option payoff function $x \mapsto(\mathcal{K}-x)^{+}$is bounded and continuous it follows that $X$ is $\mathcal{F}_{T^{-}}$ measurable and in $\mathbb{L}^{2}\left(\mathbb{F}_{+}\right)$. Therefore, the conditions of Theorem 1 are satisfied, yielding that the worst-case good-deal bound $\pi^{u}(X)$ coincides with the $Y$-component of the solution of the 2BSDE (41). In Lemma 3 below, we will express the solution to the 2BSDE (41) in terms of the classical solution $v \in \mathcal{C}^{1,2}([0, T) \times(0, \infty))$ to the Black-Scholes' type PDE

$$
\frac{\partial v}{\partial t}+\left(\gamma-h \beta \sqrt{1-\rho^{2}} \sqrt{\bar{a}_{2}}\right) x \frac{\partial v}{\partial x}+\frac{1}{2} \beta^{2}\left(\rho^{2} \bar{a}_{1}+\left(1-\rho^{2}\right) \bar{a}_{2}\right) x^{2} \frac{\partial^{2} v}{\partial x^{2}}=0
$$

on the set $[0, T) \times(0, \infty)$, with boundary condition $v(T, \cdot)=(\mathcal{K}-\cdot)^{+}$. To this end, let $P^{\bar{a}}=P^{0} \circ\left(\bar{a}^{1 / 2} B\right)^{-1} \in \mathcal{P}_{[\underline{a}, \bar{a}]}$ such that $\langle B\rangle_{t}=\bar{a} t P^{\bar{a}} \otimes d t$-a.s.. The process $L$ under $P^{\bar{a}}$ is a geometric Brownian motion with dynamics

$$
d L_{t}=L_{t}\left(\gamma d t+\bar{\beta}\left(\bar{\rho} d W_{t}^{1, P^{\bar{a}}}+\sqrt{1-\bar{\rho}^{2}} d W_{t}^{2, P^{\bar{a}}}\right)\right), t \in[0, T], P^{\bar{a}} \text {-a.s. }
$$

where $W^{P^{\bar{a}}}=\left(W^{1, P^{\bar{a}}}, W^{2, P^{\bar{a}}}\right):=(\bar{a})^{-1 / 2} B$ is a $P^{\bar{a}}$-Brownian motion and

$\bar{\beta}:=\beta\left(\rho^{2} \bar{a}_{1}+\left(1-\rho^{2}\right) \bar{a}_{2}\right)^{\frac{1}{2}}>0$ and $\bar{\rho}:=\rho \sqrt{\bar{a}_{1}}\left(\rho^{2} \bar{a}_{1}+\left(1-\rho^{2}\right) \bar{a}_{2}\right)^{-\frac{1}{2}} \in[-1,1]$.

Hence a closed-form expression for $v\left(t, L_{t}\right)$, with $v$ being the solution to the PDE (43), is given by the Black-Scholes formula for the price of the put option $X=(\mathcal{K}-$ $\left.L_{T}\right)^{+}$in the model $P^{\bar{a}}$. By arguments analogous to the derivations of the formulas in (Becherer and Kentia 2017, Section 3.2.1), $v\left(t, L_{t}\right)$ coincides $P^{\bar{a}}$-a.s. with the valuation bound $\pi_{t}^{u, P^{\bar{a}}}(X)$ for all $t \leq T$, and is given in closed form as

$$
\begin{aligned}
v\left(t, L_{t}\right) & =\pi_{t}^{u, P^{\bar{a}}}(X)=\mathcal{K} N\left(-d_{-}\right)-L_{t} e^{m(T-t)} N\left(-d_{+}\right) \\
& \left.=e^{m(T-t)} * \mathrm{~B} / \mathrm{S} \text {-put-price (time: } t \text {, spot: } L_{t}, \text { strike: } \mathcal{K} e^{-m(T-t)} \text {, vol: } \bar{\beta}\right),
\end{aligned}
$$

where "B/S-put-price" denotes the standard Black-Scholes pricing formula with zero interest rate, "vol" abbreviating volatility, $N$ denoting the cdf of the standard normal distribution, $m:=\gamma-h \bar{\beta} \sqrt{1-\bar{\rho}^{2}}=\gamma-h \beta \sqrt{1-\rho^{2}} \sqrt{\bar{a}_{2}}$, and $d_{ \pm}:=\left(\ln \left(L_{t} / \mathcal{K}\right)+\left(m \pm \frac{1}{2} \bar{\beta}^{2}\right)(T-t)\right)(\bar{\beta} \sqrt{T-t})^{-1}$. The details of the proof for the following lemma can be found in the Appendix.

Lemma 3 The triple $(Y, Z, K)$ with $Y_{t}=v\left(t, L_{t}\right), \quad Z_{t}=$ $\beta L_{t} \frac{\partial v}{\partial x}\left(t, L_{t}\right)\left(\rho, \sqrt{1-\rho^{2}}\right)^{t r}$, and $K$ given by $(50)$ for $v \in \mathcal{C}^{1,2}([0, T) \times(0, \infty))$ being the solution to the PDE (43) satisfies $(Y, Z) \in \mathbb{D}^{2}\left(\mathbb{F}^{\mathcal{P}_{[\underline{a}, \bar{a}]}}\right) \times \mathbb{H}^{2}\left(\mathbb{F}^{\mathcal{P}_{[\underline{a}, \bar{a}]}}\right)$ 
and is the unique solution to the 2BSDE (41). In particular, the stochastic integral $\int_{0}^{\cdot} Z_{s}^{t r} d B_{s}$ can be defined pathwise.

Worst-case model for valuation and hedging: Using Lemma 3, Theorem 1 implies by (44) that the robust good-deal bound for a put option $X=\left(\mathcal{K}-L_{T}\right)^{+}$is given in closed-form by

$$
\pi_{t}^{u}(X)=\mathcal{K} N\left(-d_{-}\right)-L_{t} e^{m(T-t)} N\left(-d_{+}\right), t \in[0, T], P^{\bar{a}} \text {-a.s.. }
$$

So, the robust good-deal bound $\pi^{u}(X)$ for a put option $X=\left(\mathcal{K}-L_{T}\right)^{+}$is attained at the "maximal" volatility matrix $\bar{a}$, and can be computed as in absence of uncertainty, but in a worst-case model $P^{\bar{a}} \in \mathcal{P}_{[a, \bar{a}]}$ in which $\langle B\rangle_{t}=\bar{a} t$ holds $P^{\bar{a}} \otimes d t$-a.e., yielding $\pi_{t}^{u}(X)=\pi_{t}^{u, P^{\bar{a}}}(X), t \leq T, P^{\bar{a}}$-a.s.. In addition, $\pi_{t}^{u}(X)$ is given explicitly in terms of a Black-Scholes' type formula, for modified strike $\mathcal{K} \exp (-m(T-t))$ and volatility $\bar{\beta}=\beta\left(\rho^{2} \bar{a}_{1}+\left(1-\rho^{2}\right) \bar{a}_{2}\right)^{1 / 2}$. Theorem 2 and Lemma 3 imply by (49) that the robust good-deal hedging strategy $\bar{\phi}:=\bar{\phi}(X)$ is

$$
\begin{aligned}
\bar{\phi}_{t} & =-\beta e^{m(T-t)} N\left(-d_{+}\right) L_{t} \widehat{a}_{t}^{-1 / 2} \widehat{\Pi}_{t}\left(\widehat{a}_{t}^{1 / 2}\left(\rho, \sqrt{1-\rho^{2}}\right)^{\operatorname{tr}}\right) \\
& =-\beta e^{m(T-t)} N\left(-d_{+}\right) L_{t}\left(\rho+\frac{\widehat{a}_{t}^{12}}{\widehat{a}_{t}^{11}} \sqrt{1-\rho^{2}}, 0\right)^{\operatorname{tr}}, \quad P^{\bar{a}} \otimes d t \text {-a.e., }
\end{aligned}
$$

where we have used the fact that $\widehat{a}^{-1 / 2} \widehat{\Pi}\left(\widehat{a}^{1 / 2} z\right)=\left(z_{1}+\frac{\widehat{a}^{12}}{\widehat{a}^{11}} z_{2}, 0\right)^{\operatorname{tr}}$ for $z=$ $\left(z_{1}, z_{2}\right)^{\text {tr }} \in \mathbb{R}^{2}$, which is straightforward by (40) and (39). Analogously the lower good-deal bound $\pi^{l}(X)$ and corresponding hedging strategy can also be computed, but under the worst-case measure $P^{\underline{a}} \in \mathcal{P}_{[\underline{a}, \bar{a}]}$ for "minimal" volatility matrix $\underline{a}$.

Comparison with robust superreplication under volatility uncertainty: Intuitively, as the bound $h$ on the Sharpe ratios increases to infinity, the (upper) good-deal bound $\pi^{u}(X)$ should increase towards the robust upper no-arbitrage valuation bound, studied in Avellaneda et al. (1995); Lyons (1995); Denis and Martini (2006); Vorbrink (2014); Nutz and Soner (2012); Neufeld and Nutz (2013). Our message from this example is in accordance with Avellaneda et al. (1995); Lyons (1995); El Karoui et al. (1998); Epstein and Ji (2013); Vorbrink (2014) who showed that under ambiguous volatility, Black-Scholes valuation and hedging of vanilla put (or call) options under maximal (resp., minimal) volatility corresponds to the worst-case for the seller (resp., for the buyer). Instead of superreplication we focus here on robust good-deal hedging under volatility uncertainty for valuation at the worst-case good-deal bound. Beyond some simplifications (particular payoff function) in comparison to Avellaneda et al. (1995); El Karoui et al. (1998); Vorbrink (2014), our setup also includes some more original aspects: We study an option on a non-tradable asset $L$ with possibly nonperfect correlation with $S$, and there is incompleteness in the sense that the option $X$ is already clearly non-replicable by dynamic trading under any individual prior (if $|\rho|<1$ ), even without model uncertainty. Further, we now show that the robust good-deal hedging strategy $\bar{\phi}(X)$ is very different from (the risky asset component 
of) the super-replicating strategy, in general. Indeed since $0 \leq X \leq \mathcal{K}$ holds pathwise, the (upper) no-arbitrage bound (or superreplication price) process $\widehat{V}(X)$ under $P^{\bar{a}}$ defined by

$$
\widehat{V}_{t}(X):=\operatorname{ess}_{Q \in \mathcal{M}^{e}\left(P^{\bar{a}}\right)}^{P^{\bar{a}}} E_{t}^{Q}[X], t \in[0, T], P^{\bar{a}} \text {-a.s. }
$$

satisfies $\pi_{t}^{u, P^{\bar{a}}}(X ; h) \leq \widehat{V}_{t}(X) \leq \mathcal{K}, P^{\bar{a}}$-a.s., for $\pi_{t}^{u, P^{\bar{a}}}(X ; h)$ denoting the gooddeal bound in the model $P^{\bar{a}}$ with Sharpe ratio constraint $h \in[0, \infty)$ and being given by (44) for arbitrary but fixed $h$. If $|\rho|<1$, then $\pi_{t}^{u, P^{\bar{a}}}(X ; h)$ for $t<T$ increases to $\mathcal{K}$ as $h$ tends to $+\infty$ (since $m \rightarrow-\infty, d_{ \pm} \rightarrow-\infty$ ), and hence we obtain, very differently from the good deal bound from (45), that

$$
\widehat{V}_{t}(X)=\mathcal{K} \mathbb{1}_{\{t<T\}}+X \mathbb{1}_{\{t=T\}}, t \in[0, T], P^{\bar{a}} \text {-a.s.. }
$$

The superreplication price $\widehat{V}(X)$ has the optional decomposition (cf. Kramkov 1996, Theorem 3.2)

$$
\widehat{V}_{t}(X)=\widehat{V}_{0}(X)+\int_{0}^{t} \widehat{\phi}_{s} d B_{s}^{1}-\widehat{C}_{t}, t \in[0, T], P^{\bar{a}} \text {-a.s. },
$$

where $\int_{0}^{\cdot} \widehat{\phi}_{s} d B_{s}^{1}$ and $\widehat{C}$ are unique (see Kramkov 1996, Theorem 2.1 and Lemma 2.1). One obtains by (47) that $\int_{0}^{\cdot} \widehat{\phi}_{s} d B_{s}^{1}=0$ and $\widehat{C}=(\mathcal{K}-X) \mathbb{1}_{\{T\}}$. Note from (46) that $\bar{\phi}=\left(Z^{1}, 0\right)^{\text {tr }} P^{\bar{a}} \otimes d t$-a.e. since $\widehat{a}=\bar{a} P^{\bar{a}} \otimes d t$-a.e.. For $\rho \neq 0$, the process $Z^{1}$ is non-trivial under $P^{\bar{a}} \otimes d t$. Overall for $0<|\rho|<1, \int_{0}^{*} \bar{\phi}_{s}^{\mathrm{tr}} d B_{s}=\int_{0}^{r} Z_{s}^{1} d B_{s}^{1}$ cannot be equal to $\int_{0} \widehat{\phi}_{s} d B_{s}^{1} \equiv 0 P^{\bar{a}} \otimes d t$-almost everywhere. This means that for $0<|\rho|<1$ the good-deal hedging strategy $\bar{\phi}$ for the put option on the non-traded asset is not the traded asset component of the super-replicating strategy for the option in the model $P^{\bar{a}}$. So this example clearly demonstrates that the robust good-deal hedging strategy $\bar{\phi}$ is in general very different from a super-replicating strategy under volatility uncertainty for the set $\mathcal{P}_{[\underline{a}, \bar{a}]}$ of reference priors. Notice in particular that $\pi_{t}^{u}(X)+\int_{t}^{T} \bar{\phi}_{s}^{\mathrm{tr}} d B_{s}$ does not dominate the claim $X P^{\bar{a}}$-almost surely, and hence in particularly not $\mathcal{P}_{[\underline{a}, \bar{a}]}$-quasi-surely.

\section{Case with combined drift and volatility uncertainty}

The approach of Section "Uncertainty solely about the volatility" to derive closedform valuations and hedges may not work in general when there is drift uncertainty in addition to volatility uncertainty. Indeed one would need, by the preceding valuation and hedging Theorems 1 and 2, first to identify a candidate worst-case drift parame$\operatorname{ter} \bar{\theta}$ (and then possibly a worst-case volatility) satisfying (36). This requires finding a minimizer over $\theta \in \boldsymbol{\Theta} \equiv\left\{x \in \mathbb{R}^{2}:|x| \leq \delta\right\}$ of the quantities $\widehat{F}^{\theta}\left(\widehat{a}^{1 / 2} Z\right)$ given by (20) for the $Z$-component of the solution to the 2BSDE (28). However, it is not clear at all how this could be done in general (or even explicitly), given that the expression for $\widehat{F}^{\theta}$ is quite complex by non-triviality of the kernel $\operatorname{Ker}\left(\sigma \widehat{a}^{1 / 2}\right)$. Note that the latter occurs because of the singularity of the volatility matrices $\sigma_{t} \in \mathbb{R}^{d \times n}$ when $d<n$, i.e., under market incompleteness under each prior. Furthermore, even if one could 
identify $\bar{\theta}$, the complexity of the formula for $\widehat{F}^{\bar{\theta}}\left(\widehat{a}^{1 / 2} Z\right)=\widehat{F}\left(\widehat{a}^{1 / 2} Z\right)$ makes it very difficult to derive the corresponding worst-case volatility. This issue does not appear in the less general setting of Section "Uncertainty solely about the volatility" where, thanks to the zero drift assumption $b=0$ for the traded asset $S$, the expression for $\widehat{F}\left(\widehat{a}^{1 / 2} Z\right)$ greatly simplifies to (42) and this allows by direct comparison to obtain $\bar{a}$ as the corresponding worst-case volatility. If market incompleteness is mainly due to the presence of volatility uncertainty (i.e., market is complete under every prior, and hence $\operatorname{Ker}\left(\sigma \widehat{a}^{1 / 2}\right)$ is trivial), then drift uncertainty is redundant as it does not have influence on (essentially superreplication) valuation bounds (and respective strategies) for any contingent claim, as $\widehat{F}^{\theta}(z)=\widehat{F}(z)=\widehat{\xi}^{\mathrm{tr}} z$ for any $\theta \in \boldsymbol{\Theta}, z \in \mathbb{R}^{n}$. This has been argued in more detail by (Epstein and Ji 2013, Example 3) and is perhaps not surprising. Indeed, it is well-known that the Black-Scholes price does not depend on the drift of the underlying and consequently remains unaffected by drift uncertainty. Superreplication (q.s.) appears as a natural notion for robust hedging under market completeness for every prior and is well-studied in the literature (cf. Avellaneda et al. 1995; Lyons 1995; Nutz and Soner 2012; Neufeld and Nutz 2013; Epstein and Ji 2013; Vorbrink 2014), where for the above reason it is standard to assume zero drift. However, for incomplete markets (i.e., for $d<n$ ), combined uncertainty on drifts and volatilities becomes relevant for related approaches to valuation and hedging, that are less expensive than superreplication. One may ask, whether one could identify worst-case drifts and volatilities explicitly for certain examples. Yet, even for a vanilla put option as in Section "Uncertainty solely about the volatility", we are not aware of a closed-form solution for this. Already in the case of non-zero drift $b$, which is not even subject to uncertainty, we admit that we are not able to state a worst-case volatility in closed form (e.g., by identifying it as $\bar{a}$ like we did in Section "Uncertainty solely about the volatility"). To demonstrate why this case is effectively already less tractable, let us assume for simplicity the following model parameters: $\sigma^{S}=1, \gamma=0, \beta=1, \delta=0$ and $\rho=0$. The dynamics for $S$ and $L$ are then

$$
d S_{t}=S_{t}\left(b d t+d B_{t}^{1}\right) \quad \text { and } \quad d L_{t}=L_{t} d B_{t}^{2}, \quad \mathcal{P}_{[\underline{a}, \bar{a}]^{- \text {-q.s. }} .}
$$

Here, $\widehat{\xi}=\left(b \widehat{c}^{11} / \widehat{a}^{11}, b \widehat{c}^{12} / \widehat{a}^{11}\right)^{\operatorname{tr}}, \quad \operatorname{Ker}\left(\sigma \widehat{a}^{1 / 2}\right)=\operatorname{Span}\{\widehat{\eta}\}$ with $\widehat{\eta}:=$ $\left(-\widehat{c}^{12} / \widehat{c}^{11}, 1\right)^{\mathrm{tr}}$. By (14) for $P \in \mathcal{P}_{[\underline{a}, \bar{a}]}$, the set $\mathcal{Q}^{\text {ngd }}(P)$ consists of all measures $Q^{\varepsilon}$ with $d Q^{\varepsilon}={ }^{(P)} \mathcal{E}\left((-\widehat{\xi}+\varepsilon \widehat{\eta}) \cdot W^{P}\right) d P$, for $\mathbb{R}$-valued processes $\varepsilon$ with $|\varepsilon| \leq \widehat{c}^{11}\left(\widehat{a}^{11}\right)^{-1 / 2}\left(h^{2}-b^{2} / \widehat{a}^{11}\right)^{1 / 2}$ everywhere. Since $B^{2}=\left(\widehat{c}^{12}, \widehat{c}^{22}\right) \cdot W^{P}$ and $\left(\widehat{c}^{12}\right)^{2}+\left(\widehat{c}^{22}\right)^{2}=\widehat{a}^{22}$, then a change of measure from $P$ to $Q^{\varepsilon}$ yields

$d L_{t}=L_{t}\left(\widehat{a}_{t}^{22} d W_{t}^{\varepsilon}-\left(b \widehat{a}_{t}^{12}\left(\widehat{a}_{t}^{11}\right)^{-1}-\varepsilon_{t}\left(\widehat{c}_{t}^{11}\right)^{-1}\left(\widehat{a}_{t}^{11} \widehat{a}_{t}^{22}-\left(\widehat{a}_{t}^{12}\right)^{2}\right)^{1 / 2}\right) d t\right) \quad Q^{\varepsilon}$-a.s.

where $W^{\varepsilon}$ is a one-dimensional $Q^{\varepsilon}$-Brownian motion. As the put payoff function $(\mathcal{K}-\cdot)^{+}$is non-increasing, the good-deal bound under $P$ at time zero $\pi_{0}^{u, P}((\mathcal{K}-$ $\left.\left.L_{T}\right)^{+}\right)=\sup _{\varepsilon} E^{Q^{\varepsilon}}\left[\left(\mathcal{K}-L_{T}\right)^{+}\right]$is then attained at the smallest possible $\varepsilon$ which is $-\widehat{c}^{11}\left(\widehat{a}^{11}\right)^{-1 / 2}\left(h^{2}-b^{2} / \widehat{a}^{11}\right)^{1 / 2}$. After replacing in the dynamics of $L$ and taking 
the supremum over all $P \in \mathcal{P}_{[\underline{a}, \bar{a}]}$, we obtain by Part 2 of Remark 12 that the robust good-deal bound at time $t=0$ is

$$
\pi_{0}^{u}(X)=\sup _{a \in \mathbb{S}_{2}^{>0} \cap[\underline{a}, \bar{a}]} E^{P^{0}}\left[\left(\mathcal{K}-L_{T}^{\gamma(a), \beta(a)}\right)^{+}\right],
$$

wit $\beta(a):=a^{22}, \gamma(a):=-b\left(a^{12} / a^{11}\right)-\left(h^{2}-b^{2} / a^{11}\right)^{\frac{1}{2}}\left(a^{11} a^{22}-\left(a^{12}\right)^{2}\right)^{\frac{1}{2}}\left(a^{11}\right)^{-\frac{1}{2}}$ and for $L^{\gamma, \beta}$ having dynamics $\left.d L_{t}^{\gamma, \beta}=L_{t}^{\gamma, \beta}\left(\gamma_{t} d t+\beta_{t} d W_{t}\right)\right), t \in[0, T]$, with $W$ being a one-dimensional $P^{0}$-Brownian motion. Hence, we recognize that (48) is given by the value of a standard Markovian optimal control problem with state process $L^{\gamma, \beta}$ but with control strategies $\left(\gamma_{t}, \beta_{t}\right)_{t \in[0, T]}$ taking values in the set

$$
\left\{(\gamma, \beta) \in \mathbb{R}^{2} \mid \beta=\beta(a) \text { and } \gamma=\gamma(a) \text { for some } a \in \mathbb{S}_{2}^{>0} \cap[\underline{a}, \bar{a}]\right\},
$$

that is, in general, not rectangular, what clearly complicates the derivation of optimal controls. The payoff function $x \mapsto(\mathcal{K}-x)^{+}$being non-increasing and convex, clearly a volatility matrix $a^{*}$ which simultaneously maximizes $a \mapsto \beta(a)$ and minimizes $a \mapsto \gamma(a)$ would be an optimizer for (48). In the particular case where $b=0$, one can check that $a^{*}$ equals $\bar{a}$, yielding (again) the closed-form solution of Section "Uncertainty solely about the volatility". Yet, when $b \neq 0$, it gets more complex to describe $a^{*}$.

This complexity should not be attributed to ambiguity of volatility per se. Indeed, Section "Uncertainty solely about the volatility" here reveals a closed-form solution for the problem of our case study and the characterization for the general case by our main theorems is given by 2BDSE, whose generator may be shown to be convex (cf. Remark 12). Likewise, for uncertainty solely about drifts, there exist a simple closedform solution for the case study problem and a full characterization by standard BSDEs for the general one, see Becherer and Kentia (2017). The higher complexity, noted above, thus appears as a phenomenon that is to be attributed to the combined uncertainty.

\section{Sensitivity with respect to drift parameter of non-traded asset}

Despite the lack of closed-form expression for valuations under combined uncertainties, still, some further insight can be obtained by investigating simply the sensitivity of the robust good-deal bound $\pi^{u}(X)=: \pi^{u}(X ; \gamma)$ with respect to a (fixed constant) drift $\gamma$ of the non-traded asset $L$, solely under volatility uncertainty. This is straightforward, by exploiting the explicit formulas obtained in the Section "Uncertainty solely about the volatility". By (45), one gets for any $t \in[0, T]$ and $\gamma \in \mathbb{R}$ that $\pi_{t}^{u}(X ; \gamma)=e^{(T-t) m(\gamma)} \pi_{t}^{B S}(X ; \gamma), P^{\bar{a}}$-a.s., where $\pi_{t}^{B S}(X ; \gamma)$ denotes the Black-Scholes price at time $t$ for the put option $X=\left(\mathcal{K}-L_{T}\right)^{+}$on underlying $L$ with volatility $\bar{\beta}$ under $P^{\bar{a}}$, for risk-free rate $m(\gamma):=\gamma-h \beta \sqrt{1-\rho^{2}} \bar{a}_{2}$. Hence, differentiating $\pi_{t}^{u}(X): \gamma \mapsto \pi_{t}^{u}(X ; \gamma)$ gives

$\frac{\partial \pi_{t}^{u}}{\partial \gamma}=(T-t) e^{(T-t) m(\gamma)} \pi_{t}^{B S}(\gamma)-\mathcal{K}(T-t) N\left(-d_{-}\right)=-(T-t) e^{(T-t) m(\gamma)} L_{t} N\left(-d_{-}\right)$. 
Since the far right side is always non-positive, then $\frac{\partial \pi_{t}^{u}(X)}{\partial \gamma} \leq 0$. Therefore, we obtain what intuition suggests: The robust good-deal bound $\pi^{u}(X ; \gamma)$ for the European put option $X=\left(\mathcal{K}-L_{T}\right)^{+}$is non-increasing in $\gamma$ for the model $P^{\bar{a}}$. This implies that for $\gamma, \bar{\gamma} \in \mathbb{R}$ specifying an interval range $[\gamma, \bar{\gamma}]$ for the (constant) drift parameter of $L$, the worst-case drift corresponds to $\underline{\gamma}$, and is that for which the supremum ess $\sup _{\gamma \in[\gamma, \bar{\gamma}]}^{P^{\bar{a}}} \pi_{t}^{u}(X ; \gamma)$ is attained for any $t \in[0, T]$. Note that this supremum may be different from the worst-case good-deal bound (defined in (21) and characterized by Theorem 1) under combined drift and volatility uncertainties, as the latter parametrizes drift uncertainty instead in terms of stochastic drifts $\gamma$ that satisfy $\gamma \leq \gamma \leq \bar{\gamma}$ pointwise and there is no apparent reason why the worst-case volatility for every fixed (stochastic) drift $\gamma$ should be $\bar{a}$.

\section{Appendix}

This section contains some proofs and details omitted in the main body of the paper. This includes Lemma 4 which is used in the proof of Theorem 2.

Proof (of Proposition 1) Consider the family $(\mathcal{P}(t, \omega))_{(t, \omega) \in[0, T] \times \boldsymbol{\Omega}}$ of sets probability measures given by $\mathcal{P}(t, \omega):=\mathcal{P}_{[\underline{a}, \bar{a}]}$ for all $(t, \omega) \in[0, T] \times$ $\boldsymbol{\Omega}$ and the constant set-valued process $\mathrm{D}:[0, T] \times \boldsymbol{\Omega} \rightarrow \mathbb{S}_{n}^{>0}$ with $\mathrm{D}_{t}(\omega):=[\underline{a}, \bar{a}]$ for all $(t, \omega) \in[0, T] \times \boldsymbol{\Omega}$. Clearly, $\mathrm{D}$ has the properties required in (Neufeld and Nutz 2013, Example 2.1) from which the first claim of (Neufeld and Nutz 2013, Cor. 2.6) implies that the constant family $\mathcal{P}(t, \omega) \equiv \mathcal{P}_{[\underline{a}, \bar{a}]}$ satisfies Condition A therein, hence in particular the measurability and stability conditions of Assumption 2.1 (iii)-(v) of Possamaï et al. (2018), whereby (iii) in particular follows from (Neufeld and Nutz 2013, Condition (A1)) since a countable product of analytic sets is again analytic (Bertsekas and Shreve 1978, Proposition 7.38). This together with our Assumption 1 and $X \in \mathbb{L}^{2}\left(\mathbb{F}_{+}\right)$ imply Assumptions 2.1 and 3.1 of Possamaï et al. (2018) from which a direct application of Theorems 4.1 and 4.2 therein yields existence and uniqueness of a 2BSDE solution $\left(Y, Z,\left(K^{P}\right)_{P \in \mathcal{P}_{[\underline{a}, \bar{a}]}}\right) \in \mathbb{D}^{2}\left(\mathbb{F}_{+}^{\mathcal{P}_{[\underline{a}, \bar{a}]}}\right) \times \mathbb{H}^{2}\left(\mathbb{F}^{\mathcal{P}_{[\underline{a}, \bar{a}]}}\right) \times \mathbb{I}^{2}\left(\left(\mathbb{F}_{+}^{P}\right)_{P \in \mathcal{P}_{[\underline{a}, \bar{a}]}}\right)$ satisfying the representation (4), where $\mathbb{F}_{+}^{\mathcal{P}_{[\underline{a}, \bar{a}]}}=\left(\mathcal{F}_{t+}^{\mathcal{P}_{[\underline{a}, \bar{a}]}}\right)_{t \in[0, T]}$, with $\mathcal{F}_{t+}^{\mathcal{P}_{[\underline{a}, \bar{a}]}}:=$ $\bigcap_{P \in \mathcal{P}_{[\underline{a}, \bar{a}]}} \mathcal{F}_{t+}^{P}$. Note that the additional orthogonal martingale component in the 2BSDE formulation of Possamaï et al. (2018) vanishes in this case thanks to the martingale representation property of $\mathcal{P}_{[\underline{a}, \bar{a}]}$ in Lemma 1 . Moreover, since by Lemma 1 the filtration $\mathbb{F}^{P}$ is actually right continuous for every $P \in \mathcal{P}_{[\underline{a}, \bar{a}]}$, it follows that $\mathcal{F}_{t+}^{P}=\mathcal{F}_{t}^{P}$ for $t \in[0, T], P \in \mathcal{P}_{[a, \bar{a}]}$ which implies that the minimum condition in the definition of a 2BSDE solution in (Possamaï et al. 2018, Definition 4.1) is equivalent to (3). In particular, we have $\mathbb{F}_{+}^{\mathcal{P}_{[\underline{a}, \bar{a}]}}=\mathbb{F}^{\mathcal{P}_{[\underline{a}, \bar{a}]}}$.

Proof (of Proposition 4) By the classical comparison theorem for standard BSDEs, one easily sees for every $P \in \mathcal{P}_{[a, \bar{a}]}$ that $\widetilde{\mathcal{Y}}^{P, X}=\rho^{P}(X)$, where $\left(\tilde{\mathcal{Y}}^{P, X}, \widetilde{\mathcal{Z}}^{P, X}\right)$ is the 
unique solution to the standard Lipschitz BSDE under $P$ with data $\left(-\widehat{F}^{\prime}\left(\widehat{a}^{\frac{1}{2}} \cdot\right), X\right)$ for $F^{\prime}$ given by (31). In addition, one can verify as in the proof of Theorem 1 for $(F, X)$ that $\left(F^{\prime}, X\right)$ satisfies Assumption 1 as well. The required result then follows from an application of Proposition 1.

Proof (of Lemma 3) By Theorem 1 the 2BSDE (41) admits a unique solution which remains to be identified as claimed. For any $P \in \mathcal{P}_{[\underline{a}, \bar{a}]}$, Itô's formula and (43) yield for $t \in[0, T], P$-a.s., that

$v\left(t, L_{t}\right)=X-\int_{t}^{T} Z_{s}^{\mathrm{tr}} d B_{s}+h \int_{t}^{T}\left(\widehat{a}_{s}^{11} \widehat{a}_{s}^{22}-\left(\widehat{a}_{s}^{12}\right)^{2}\right)^{1 / 2}\left(\widehat{a}_{s}^{11}\right)^{-1 / 2}\left|Z_{s}^{2}\right| d s+K_{T}-K_{t}$,

where, by using (44), the processes $Z=\left(Z^{1}, Z^{2}\right)^{\operatorname{tr}}$ and $K$ are given by

$$
\begin{aligned}
Z_{t}= & \beta L_{t} \frac{\partial v}{\partial x}\left(t, L_{t}\right)\left(\rho, \sqrt{1-\rho^{2}}\right)^{\operatorname{tr}}=-\beta e^{m(T-t)} N\left(-d_{+}\right) L_{t}\left(\rho, \sqrt{1-\rho^{2}}\right)^{\operatorname{tr}},(49) \\
K_{t}= & \int_{0}^{t}\left[h \beta \sqrt{1-\rho^{2}} L_{s} \frac{\partial v}{\partial x}\left(s, L_{s}\right)\left(\left(\widehat{a}_{s}^{11} \widehat{a}_{s}^{22}-\left(\widehat{a}_{s}^{12}\right)^{2}\right)^{1 / 2}\left(\widehat{a}_{s}^{11}\right)^{-1 / 2}-\sqrt{\bar{a}_{2}}\right)(50)\right. \\
& \left.+\frac{1}{2} \beta^{2} L_{s}^{2} \frac{\partial^{2} v}{\partial x^{2}}\left(s, L_{s}\right)\left(\rho^{2}\left(\bar{a}_{1}-\widehat{a}_{s}^{11}\right)+\left(1-\rho^{2}\right)\left(\bar{a}_{2}-\widehat{a}_{s}^{22}\right)-2 \rho \sqrt{1-\rho} \widehat{a}_{s}^{12}\right)\right] d s .
\end{aligned}
$$

To show that $K$ is a non-decreasing process, notice that $\widehat{a} \leq \bar{a} P \otimes d t$-a.e. yields $\widehat{a}^{1 / 2} \leq \bar{a}^{1 / 2} P \otimes d t$-a.e. and both inequalities imply that $P \otimes d t$-a.e. $\left(\widehat{a}^{11} \widehat{a}^{22}-\left(\widehat{a}^{12}\right)^{2}\right)^{1 / 2}\left(\widehat{a}^{11}\right)^{-1 / 2} \leq\left(\widehat{a}^{22}\right)^{1 / 2} \leq \sqrt{\bar{a}_{2}}$ and

$$
\begin{aligned}
& \rho^{2}\left(\bar{a}_{1}-\widehat{a}^{11}\right)+\left(1-\rho^{2}\right)\left(\bar{a}_{2}-\widehat{a}^{22}\right)-2 \rho \sqrt{1-\rho} \widehat{a}^{12} \\
& \quad=\left(\rho, \sqrt{1-\rho^{2}}\right) \bar{a}\left(\rho, \sqrt{1-\rho^{2}}\right)^{\operatorname{tr}}-\left(\rho, \sqrt{1-\rho^{2}}\right) \widehat{a}\left(\rho, \sqrt{1-\rho^{2}}\right)^{\operatorname{tr}} \geq 0 .
\end{aligned}
$$

Thus, the process $K$ is $P$-a.s. non-decreasing because the delta of the put option in the Black-Scholes model is non-positive and the gamma is non-negative, i.e., $\frac{\partial v}{\partial x}\left(t, L_{t}\right) \leq 0$ and $\frac{\partial^{2} v}{\partial x^{2}}\left(t, L_{t}\right) \geq 0$ for all $t \in[0, T]$ using (44). Moreover, it can be shown following arguments analogous to those in the proof of (Soner et al. 2012, Theorem 5.3) that the process $K$ satisfies the minimum condition (3); we omit the essentially technical details which we refer to (Kentia 2015, Section 4.3.3, proof of Lemma 4.25).

It remains to show that $v(\cdot, L.) \in \mathbb{D}^{2}\left(\mathbb{F}^{\mathcal{P}_{[\underline{a}, \bar{a}]}}\right), Z \in \mathbb{H}^{2}\left(\mathbb{F}^{\mathcal{P}_{[\underline{a}, \bar{a}]}}\right)$ and that the stochastic integral $\int_{0}^{\cdot} Z_{s}^{\mathrm{tr}} d B_{s}$ can be constructed pathwise. This will conclude by uniqueness of the solution to the $2 \mathrm{BSDE}$ that $(v(\cdot, L), Z, K$.$) is the unique solu-$ tion to the 2BSDE (41) for $Z$ given as in (49) and $K$ as in (50). Since $v$ is $\mathcal{C}^{1,2}$

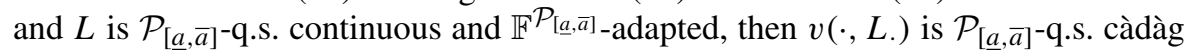
and $\mathbb{F}^{\left.\mathcal{P}_{[a,}, \bar{a}\right]}$-progressively measurable and $Z$ is $\mathbb{F}^{\left.\mathcal{P}_{[a,}, \bar{a}\right]}$-predictable. That $v(\cdot, L$.$) is in$ $\mathbb{D}^{2}\left(\mathbb{F}^{\left.\mathcal{P}_{[\underline{a}, \bar{a}]}\right)}\right.$ now follows from (44) which indeed implies $0 \leq v\left(t, L_{t}\right) \leq \mathcal{K}$ pathwise. By (49) and since $\underline{a} \leq \widehat{a} \leq \bar{a}$ holds $P \otimes d t$-a.e. for any $P \in \mathcal{P}_{[\underline{a}, \bar{a}]}$, one has 
$\left|\widehat{a}_{t}^{1 / 2} Z_{t}\right|^{2} \leq \max \left(\bar{a}_{1}, \bar{a}_{2}\right) \beta^{2} e^{2|m| T} L_{t}^{2} P \otimes d t$-a.e. for any $P \in \mathcal{P}_{[\underline{a}, \bar{a}]}$. Hence, to conclude that $Z \in \mathbb{H}^{2}\left(\mathbb{F}^{\mathcal{P}_{[\underline{a}, \bar{a}]}}\right)$ it suffices to show that $\sup _{P \in \mathcal{P}_{[\underline{a}, \bar{a}]}} E^{P}\left[\int_{0}^{T} L_{t}^{2} d t\right]<\infty$. To this end, note that for any $P \in \mathcal{P}_{[\underline{a}, \bar{a}]}$ hold

$$
\int_{0}^{T} L_{t}^{2} d t \leq \beta^{-2}\left(\min \left(\underline{a}_{1}, \underline{a}_{2}\right)\right)^{-1}\langle L\rangle_{T} \quad \text { and } \quad L_{T}^{2} \leq L_{0}^{2} e^{\left(2|\gamma|+\beta^{2} \max \left(\bar{a}_{1}, \bar{a}_{2}\right)\right) T} \tilde{L}_{T}
$$

$P$-almost surely, for $\tilde{L}$ satisfying $\tilde{L}=1+\int_{0}^{\cdot} 2 \tilde{L}_{s} \beta\left(\rho d B_{s}^{1}+\sqrt{1-\rho^{2}} d B_{s}^{2}\right) \mathcal{P}_{[\underline{a}, \bar{a}]^{-}}$ q.s.. Clearly $E^{P}\left[\tilde{L}_{T}\right] \leq 1$ for every $P \in \mathcal{P}_{[\underline{a}, \bar{a}]}$. Thus, taking expectations in (51) gives

$E^{P}\left[\int_{0}^{T} L_{t}^{2} d t\right] \leq \beta^{-2}\left(\min \left(\underline{a}_{1}, \underline{a}_{2}\right)\right)^{-1} L_{0}^{2} e^{\left(2|\gamma|+\beta^{2} \max \left(\bar{a}_{1}, \bar{a}_{2}\right)\right) T}, \quad$ for all $P \in \mathcal{P}_{[\underline{a}, \bar{a}]}$.

Taking the supremum over all $P \in \mathcal{P}_{[\underline{a}, \bar{a}]}$ yields $Z \in \mathbb{H}^{2}\left(\mathbb{F}^{\mathcal{P}_{[\underline{a}, \bar{a}]}}\right)$. As a consequence, $(v(\cdot, L), Z, K$.$) is the unique solution to the 2 \mathrm{BSDE}(41)$. Finally, $\int_{0}^{\cdot} Z_{s}^{\mathrm{tr}} d B_{s}$ can be constructed pathwise (Karandikar 1995) as $Z$ is continuous and $\mathbb{F}$-adapted.

Lemma 4 For $d<n$, let $\sigma \in \mathbb{R}^{d \times n}$ be of full (maximal) rank $d, h>0, z \in \mathbb{R}^{n}$, $\xi \in C:=\operatorname{Im} \sigma^{t r}$, and $U \subset \mathbb{R}^{n}$ be a convex-compact set. Let $\Pi(\cdot)$ and $\Pi^{\perp}(\cdot)$ denote the orthogonal projections onto the linear subspaces $C$ and $C^{\perp}=\operatorname{Ker} \sigma$, respectively, and let $F: \mathbb{R}^{n} \times \mathbb{R}^{n} \ni(\phi, \vartheta) \mapsto \xi^{t r} \phi-\vartheta^{\operatorname{tr}}(z-\phi)-h|z-\phi|$. Assume that $|\xi+\Pi(\vartheta)|<h$ for all $\vartheta \in U$. Then:

1. the vector $\bar{\phi}(\vartheta):=\Pi(z)+\left|\Pi^{\perp}(z)\right|\left(h^{2}-|\xi+\Pi(\vartheta)|^{2}\right)^{-1 / 2}(\xi+\Pi(\vartheta))$ is, for any $\vartheta \in \mathbb{R}^{n}$, the unique maximizer of $\phi \mapsto F(\phi, \vartheta)$ over $C$, the maximum being

$G(\vartheta):=F(\bar{\phi}(\vartheta), \vartheta)=-\Pi^{\perp}(\vartheta)^{\operatorname{tr}} \Pi^{\perp}(z)+\xi^{t r} \Pi(z)-\left(h^{2}-|\xi+\Pi(\vartheta)|^{2}\right)^{1 / 2}\left|\Pi^{\perp}(z)\right|$.

\section{The minmax identity}

$$
\inf _{\vartheta \in U} \sup _{\phi \in C} F(\phi, \vartheta)=F(\bar{\phi}(\bar{\vartheta}), \bar{\vartheta})=G(\bar{\vartheta})=\sup _{\phi \in C} \inf _{\vartheta \in U} F(\phi, \vartheta)
$$

holds, for $\bar{\phi}(\bar{\vartheta})$ being the $\phi$-component of the saddle point with $\bar{\vartheta}=$ $\operatorname{argmin}_{\vartheta \in U} G(\vartheta)$.

3. Assume $0 \in U$, then for $\bar{\vartheta}$ and $\bar{\phi}(\bar{\vartheta})$ we have $F(\bar{\phi}(\bar{\vartheta}), \bar{\vartheta})=\inf _{\vartheta \in U} F(\bar{\phi}(\bar{\vartheta}), \vartheta)$.

Proof As the proof of Part 1 is analogous to that of (Becherer 2009, Lemma 6.1) (or, in a more general ellipsoidal setup, of Becherer and Kentia 2017, Lemma 5.1), we leave the details to reader and just show Parts 2 and 3 here.

Part 2: For every $\phi \in \mathbb{R}^{n}$, the function $\vartheta \mapsto F(\phi, \vartheta)$ is convex, continuous. For every $\vartheta \in \mathbb{R}^{n}$ the function $\phi \mapsto F(\phi, \vartheta)$ is concave, continuous. As $U \subset \mathbb{R}^{n}$ is convex and compact, and $C$ is convex and closed, a minmax theorem (Ekeland and Temam 1999, Ch. VI, Proposition 2.3) applies and the minmax identity holds. Furthermore for any $\vartheta \in U$, the function $\phi \mapsto F(\phi, \vartheta)$ is strictly concave over 
$\left\{\boldsymbol{\Pi}^{\perp}(\phi)=0\right\}$ if $\boldsymbol{\Pi}^{\perp}(z) \neq 0$, and strictly concave at $\phi=z$ if $\Pi^{\perp}(z)=0$, since $|\xi+\Pi(\vartheta)|<h$. Hence, (Ekeland and Temam 1999, Ch. VI, Proposition 1.5) implies that the $\phi$-components of the saddle points are identical, in particular, to $\bar{\phi}(\bar{\vartheta})$ since indeed $(\bar{\phi}(\bar{\vartheta}), \bar{\vartheta})$ is a saddle point.

Part 3: The function $\mathbb{R}^{n} \ni \phi \mapsto \inf _{\vartheta \in U} F(\phi, \vartheta)=\xi^{\mathrm{tr}} \phi-\sup _{\vartheta \in U} \vartheta^{\operatorname{tr}}(z-\phi)-$ $h|z-\phi|$ is concave and continuous. In addition, it is also coercive on $C$, i.e., $F(\phi) \rightarrow$ $-\infty$ as $|\phi| \rightarrow+\infty$ for $\Pi^{\perp}(\phi)=0$ because $|\xi|<h$ and $\sup _{\vartheta \in U} \vartheta^{\operatorname{tr}}(z-\phi) \geq 0$ since $0 \in U$. Hence, by (Ekeland and Temam 1999, Ch. II, Proposition 1.2) there exists $\phi^{*} \in C$ such that $\sup _{\phi \in C} \inf _{\vartheta \in U} F(\phi, \vartheta)=\inf _{\vartheta \in U} F\left(\phi^{*}, \vartheta\right)$. In other words, $\phi^{*}$ is the $\phi$-component of a saddle point of $F$, with the other component being $\vartheta^{*}=$ $\operatorname{argmax}_{\vartheta \in U} \vartheta^{\text {tr }}\left(z-\phi^{*}\right)$. By Part 2, $\phi^{*}=\bar{\phi}(\bar{\vartheta})$, and Claim 3 follows.

Acknowledgements We thank the German Science Foundation, Berlin Mathematical School and RTG 1845 for support, and Xiaolu Tan for helpful discussions.

\section{Authors' contributions}

Authors contributed equally to the paper; both read and approved the final manuscript.

\section{Competing interests}

The authors declare that they have no competing interests.

\section{References}

Artzner, P, Delbaen, F, Eber, JM, Heath, D: Coherent measures of risk. Math. Finance. 9(3), 203-228 (1999)

Avellaneda, M, Levy, A, Paras, A: Pricing and hedging derivative securities in markets with uncertain volatilities. Appl. Math. Finance. 2(2), 73-88 (1995)

Barrieu, P, El Karoui, N: Pricing, hedging and optimally designing derivatives via minimization of risk measures. In: Carmona, R (ed.) Indifference Pricing: Theory and Applications, pp. 77-146. Princeton University Press, Princeton (2009)

Becherer, D: From bounds on optimal growth towards a theory of good-deal hedging. In: Albrecher, H, Runggaldier, W, Schachermayer, W (eds.) Advanced Financial Modelling, Radon Series on Computational and Applied Mathematics, vol 8, pp. 27-52. De Gruyter, Berlin (2009)

Becherer, D, Kentia, K: Hedging under generalized good-deal bounds and model uncertainty. Math. Meth. Oper. Res. 86(1), 171-214 (2017)

Bertsekas, DP, Shreve, SE: Stochastic Optimal Control: The Discrete Time Case. Academic Press, New York (1978)

Biagini, S, Pınar, MÇ: The robust Merton problem of an ambiguity averse investor. Math. Financ. Econ. 11(1), 1-24 (2017)

Biagini, S, Bouchard, B, Kardaras, C, Nutz, M: Robust fundamental theorem for continuous processes. Math. Finance. 27(4), 963-987 (2017)

Bielecki, T, Cialenco, I, Pitera, M: A survey of time consistency of dynamic risk measures and dynamic performance measures in discrete time: LM-measure perspective. Probab. Uncertain. Quant. Risk. 2:52, paper no.3 (2017). doi:10.1186/s41546-017-0012-9

Bielecki, TR, Cialenco, I, Zhang, Z: Dynamic coherent acceptability indices and their applications to finance. Math. Finance. 24(3), 411-441 (2014)

Björk, T, Slinko, I: Towards a general theory of good-deal bounds. Rev. Finance. 10(2), 221-260 (2006) 
Cerný, A, Hodges, SD: The theory of good-deal pricing in financial markets. In: Geman, H, DP M, Plinska, S, Vorst, T (eds.) Mathematical Finance - Bachelier Congress 2000, pp. 175-202. Springer, Berlin (2002)

Chen, Z, Epstein, LG: Ambiguity, risk and asset returns in continuous time. Econometrica. 70(4), 14031443 (2002)

Cochrane, J, Saá-Requejo, J: Beyond arbitrage: good deal asset price bounds in incomplete markets. J. Polit. Econ. 108(1), 79-119 (2000)

Delbaen, F: The structure of m-stable sets and in particular of the set of risk neutral measures. Séminaire de Probabilités XXXIX, Lecture Notes in Math. 1874, pp. 215-258. Springer, Berlin (2006)

Delbaen, F, Schachermayer, W: A general version of the fundamental theorem of asset pricing. Math. Ann. 300(1), 463-520 (1994)

Denis, L, Martini, C: A theoretical framework for the pricing of contingent claims in the presence of model uncertainty. Ann. Appl. Probab. 16(2), 827-852 (2006)

Denis, L, Hu, M, Peng, S: Function spaces and capacity related to a sublinear expectation: application to $G$-Brownian motion paths. Potential Anal. 34(2), 139-161 (2011)

Ekeland, I, Temam, R: Convex Analysis and Variational Problems. SIAM, Philadelphia (1999)

El Karoui, N, Jeanblanc-Picqué, M, Shreve, SE: Robustness of the Black and Scholes formula. Math. Finance. 8(2), 93-126 (1998)

Epstein, LG, Ji, S: Ambiguous volatility and asset pricing in continuous time. Rev. Financ. Stud. 26(7), 1740-1786 (2013)

Epstein, LG, Ji, S: Ambiguous volatility, possibility and utility in continuous time. J. Math. Econom. 50, 269-282 (2014)

Garlappi, L, Uppal, R, Wang, T: Portfolio selection with parameter and model uncertainty: A multi-prior approach. Rev. Financ. Stud. 20(1), 41-81 (2007)

Gilboa, I, Schmeidler, D: Maxmin expected utility with non-unique prior. J. Math. Econom. 18(2), 141153 (1989)

Hu, M, Ji, S, Peng, S, Song, Y: Backward stochastic differential equations driven by $G$-Brownian motion. Stoch. Process. Appl. 124(1), 759-784 (2014a)

$\mathrm{Hu}, \mathrm{M}, \mathrm{Ji}, \mathrm{S}$, Yang, S: A stochastic recursive optimal control problem under the G-expectation framework. Appl. Math. Optim. 70(2), 253-278 (2014b)

Karandikar, RL: On path-wise stochastic integration. Stoch. Process. Appl. 57(1), 11-18 (1995)

Kentia, K: Robust aspects of hedging and valuation in incomplete markets and related backward SDE theory. PhD thesis. Humboldt-Universität zu Berlin (2015). http://dx.doi.org/10.18452/17463

Klöppel, S, Schweizer, M: Dynamic utility-based good-deal bounds. Stat. Dec. 25(4), 285-309 (2007)

Kramkov, D: Optional decomposition of supermartingales and hedging in incomplete security markets. Probab. Theory Relat. Fields. 105(4), 459-479 (1996)

Lyons, TJ: Uncertain volatility and the risk-free synthesis of derivatives. Appl. Math. Finance. 2(2), 117133 (1995)

Madan, D, Cherny, A: Markets as a counterparty: an introduction to conic finance. Int. J. Theor. Appl. Finance. 13(8), 1149-1177 (2010)

Matoussi, A, Possamaï, D, Zhou, C: Robust utility maximization in nondominated models with 2BSDE: the uncertain volatility model. Math. Finance. 25(2), 258-287 (2015)

Neufeld, A, Nutz, M: Superreplication under volatility uncertainty for measurable claims. Electron. J. Probab. 18(48), 1-14 (2013)

Neufeld, A, Nutz, M: Robust utility maximization with Lévy processes. Forthcom. Math. Finance (2016). doi:10.1111/mafi.12139

Nutz, M: Path-wise construction of stochastic integrals. Electron. Commun. Probab. 17(24), 1-7 (2012a)

Nutz, M: A quasi-sure approach to the control of non-Markovian stochastic differential equations. Electron. J. Probab. 17(23), 1-23 (2012b)

Nutz, M, van Handel, R: Constructing sublinear expectations on path space. Stoch. Process. Appl. 123(8), 3100-3121 (2013)

Nutz, M, Soner, M: Superhedging and dynamic risk measures under volatility uncertainty. SIAM J. Control. Optim. 50(4), 2065-2089 (2012)

Øksendal, B, Sulem, A: Forward-backward stochastic differential games and stochastic control under model uncertainty. J. Optim. Theory Appl. 161(1), 22-55 (2014)

Peng, S: $G$-expectation, $G$-Brownian motion and related stochastic calculus of Itô type. Stochastic analysis and applications. The Abel symposium 2005, Abel Symposia book series, vol 2, pp. 541-567. Springer, Berlin (2007) 
Possamaï, D, Tan, X, Zhou, C: Stochastic control for a class of nonlinear kernels and applications. ArXiv e-print arXiv:1510.08439v1. To appear in Ann Prob (to be published in 2018). arxiv.org/pdf/1510.08439v1

Quenez, MC: Optimal portfolio in a multiple-priors model. In: Dalang, R, Dozzi, M, Russo, F (eds.) Seminar on Stochastic Analysis, Random Fields and Applications IV, Progress in Probability, vol 58, pp. 291-321. Birkhäuser, Basel (2004)

Rockafellar, RT: Integral functionals, normal integrands and measurable selections. In: Waelbroeck, L (ed.) Nonlinear Operators and Calculus of Variations, Lecture Notes in Mathematics 543, pp. 157207. Springer, Berlin (1976)

Rosazza Gianin, E, Sgarra, C: Acceptability indexes via $g$-expectations: an application to liquidity risk. Math. Financ. Econ. 7(4), 457-475 (2013)

Schied, A: Optimal investments for risk- and ambiguity-averse preferences: a duality approach. Finance. Stoch. 11(1), 107-129 (2007)

Schweizer, M: A guided tour through quadratic hedging approaches. In: Jouini, E, Cvitanić, J, Musiela, M (eds.) Option Pricing, Interest Rates and Risk Management, pp. 538-574. Cambridge University Press, Cambridge (2001)

Soner, HM, Touzi, N, Zhang, J: Quasi-sure stochastic analysis through aggregation. Electron. J. Probab. 16(67), 1844-1879 (2011)

Soner, HM, Touzi, N, Zhang, J: Wellposedness of second order backward SDEs. Probab. Theory Relat. Fields. 153(1-2), 149-190 (2012)

Soner, HM, Touzi, N, Zhang, J: Dual formulation of second order target problems. Ann. Appl. Probab. 23(1), 308-347 (2013)

Tevzadze, R, Toronjadze, T, Uzunashvili, T: Robust utility maximization for a diffusion market model with misspecified coefficients. Financ. Stoch. 17(3), 535-563 (2013)

Vorbrink, J: Financial markets under volatility uncertainty. J Math. Econom. 53, 64-78 (2014). special Section: Economic Theory of Bubbles (I) 